\title{
The stellar masses and specific star-formation rates of submillimetre galaxies ${ }^{\star}$
}

\author{
M. J. Michałowski ${ }^{1}$, J. S. Dunlop ${ }^{1}$, M. Cirasuolo ${ }^{1}$, J. Hjorth ${ }^{2}$, C. C. Hayward ${ }^{3}$, and D. Watson ${ }^{2}$ \\ ${ }^{1}$ SUPA $^{\star \star}$, Institute for Astronomy, University of Edinburgh, Royal Observatory, Edinburgh, EH9 3HJ, UK \\ e-mail: mm@roe.ac.uk \\ 2 Dark Cosmology Centre, Niels Bohr Institute, University of Copenhagen, Juliane Maries Vej 30, 2100 Copenhagen $\varnothing$, Denmark \\ 3 Harvard-Smithsonian Center for Astrophysics, 60 Garden St., Cambridge, MA 02138, USA
}

Received 13 December 2011 / Accepted 20 March 2012

\begin{abstract}
Establishing the stellar masses, and hence specific star-formation rates of submillimetre galaxies is crucial for determining the role of such objects in the cosmic history of galaxy/star formation. However, there is as yet no consensus over the typical stellar masses of submillimetre galaxies, as illustrated by the widely differing results reported from recent optical-infrared studies of submillimetre galaxies with spectroscopic redshifts $z \simeq 2-3$. Specifically, even for the same set of submillimetre galaxies, the reported average stellar masses have ranged over an order of magnitude, from $\simeq 5 \times 10^{10} M_{\odot}$ to $\simeq 5 \times 10^{11} M_{\odot}$. Here we study how different methods of analysis can lead to such widely varying results. We find that, contrary to recent claims in the literature, potential contamination of IRAC 3-8 $\mu \mathrm{m}$ photometry from hot dust associated with an active nucleus is not the origin of the published discrepancies in derived stellar masses. Instead, we expose in detail how inferred stellar mass depends on assumptions made in the photometric fitting, and quantify the individual and cumulative effects of different choices of initial mass function, different "brands" of evolutionary synthesis models, and different forms of assumed star-formation history. We review current observational evidence for and against these alternatives as well as clues from the hydrodynamical simulations, and conclude that, for the most justifiable choices of these model inputs, the average stellar mass of luminous $\left(S_{850} \gtrsim 5 \mathrm{mJy}\right)$ submillimetre galaxies is $\simeq 2 \times 10^{11} M_{\odot}$ to within a factor $\simeq 2$. We also check and confirm that this number is perfectly reasonable in the light of the latest measurements of the dynamical masses of these objects ( $\simeq 2-6 \times 10^{11} M_{\odot}$ from CO $(1-0)$ observations), and the evolving stellar mass function of the overall galaxy population. Galaxy stellar masses of this order imply that the average specific star-formation rate of submillimetre galaxies is comparable to that of other star-forming galaxies at $z>2$, at $2-3 \mathrm{Gyr}^{-1}$. This supports the view that, while rare outliers may be found at any stellar mass, most submillimetre galaxies simply form the top end of the "main-sequence" of star-forming galaxies at these redshifts. Conversely, this argues strongly against the viewpoint, frequently simply asserted in the literature, that submillimetre galaxies are extreme pathological objects, of little relevance in the cosmic history of star-formation.
\end{abstract}

Key words. galaxies: active - galaxies: evolution - galaxies: high-redshift - galaxies: ISM - galaxies: starburst submillimeter: galaxies

\section{Introduction}

The properties of galaxies selected at submillimetre wavelengths (SMGs) have yet to be fully understood. In particular, there is still no real consensus over their stellar masses. A robust measurement of the stellar masses of SMGs is key to addressing a number of important issues, including the evolution of the relationship between galaxy and black-hole masses (Borys et al. 2005; Hainline et al. 2011, H11 thereafter), and the contribution of SMGs to the overall history of stellar mass assembly (Michałowski et al. 2010a, M10 hereafter). Perhaps most importantly, a proper understanding of the stellar masses of SMGs is required to distinguish whether their massive dust-enshrouded star-formation rates are fuelled primarily by mergers, or by cold gas infall (Swinbank et al. 2008; Davé et al. 2010; González et al. 2011; Narayanan et al. 2010b; Ricciardelli et al. 2010; Hayward et al. 2011a,b, 2012). Unless the uncertainty in stellar masses can be reduced, it is in essence impossible to tell whether SMGs have specific star-formation rates $\left(\mathrm{sSFR}=\mathrm{SFR} / M_{*}\right.$ ) comparable

\footnotetext{
* Appendix A is available in electronic form at http: //www . aanda.org

$\star \star$ Scottish Universities Physics Alliance.
}

to those of other star-forming galaxy populations at $z>2$, or whether they are extreme outliers from the "main-sequence" of star-forming galaxies (Daddi et al. 2007; Noeske et al. 2007; González et al. 2010).

Most previous studies of the stellar masses of SMGs have concluded in favour of values in the range $\simeq 10^{11}-10^{12} M_{\odot}$ (Borys et al. 2005; Dye et al. 2008). However, two recent studies of $\simeq 70$ SMGs with spectroscopic redshifts $\simeq 2-3$ from Chapman et al. (2005) have reached apparently very different conclusions, highlighting the impact of alternative methods of analysis. Specifically, Michałowski et al. (2010a,b) found stellar masses consistent with previous studies, with a median value $M_{*}=3.5 \times 10^{11} M_{\odot}$ (see also Hatsukade et al. 2010; Ikarashi et al. 2011; Santini et al. 2010; Tamura et al. 2010), whereas Hainline et al. (2011) have reported values, based on the same SMGs, which are systematically lower by a factor of $\simeq 6$ (median $M_{*}=5-7 \times 10^{10} M_{\odot}$; see also Wardlow et al. 2011).

The primary objective of this paper is to explore the origins of this discrepancy, and to attempt to resolve it. More generally, however, we have taken this opportunity to expose in detail how the derived stellar masses (and hence sSFRs) of SMGs depend on different assumed forms of star-formation history, and 
different "brands" of evolutionary synthesis models. The use of a sample of SMGs with spectroscopic redshifts is especially useful for this purpose, since it is well known that, if only photometric redshifts are available, the errors in derived stellar masses can be dominated by the effect of uncertainties in $z_{\text {phot }}$.

The structure of this paper is as follows. In Sect. 2 we delineate the various factors and choices of analysis methods which influence the stellar mass determinations and quantify their impact on the final derived values. We also review recent evidence that informs the preferred choice of, for example, initial mass function (IMF) and evolutionary synthesis model, and conclude that for most reasonable choices of such model inputs the derived stellar masses in fact only differ by a factor $\simeq 2$. In Sect. 3 we investigate the consistency of our re-derived stellar masses with the latest measurements of the dynamical masses of these objects, the evolving stellar mass function of the overall galaxy population, and the predictions of theoretical simulations. We discuss the implications of our results on the sSFR of SMGs in Sect. 4, and close with a summary of our conclusions in Sect. 5. We use a cosmological model with $H_{0}=70 \mathrm{~km} \mathrm{~s}^{-1} \mathrm{Mpc}^{-1}$, $\Omega_{\Lambda}=0.7$ and $\Omega_{\mathrm{m}}=0.3$.

\section{Determining the stellar masses of SMGs}

\subsection{Initial mass function}

The choice of a particular IMF introduces a systematic uncertainty of a factor of $\simeq 2$ in the determination of the stellar masses $\left(M_{*}\right)$ and star-formation rates (SFRs; Erb et al. 2006). Specifically, the differences between the IMFs assumed by M10 (Salpeter 1955) and H11 (Chabrier 2003) accounts for a factor of 1.8 in the difference between the quoted stellar mass estimates.

Both top-heavy and standard IMFs have been claimed to reproduce the number counts of SMGs (Baugh et al. 2005; Fontanot et al. 2007; Hayward et al. 2011b). This confusing situation is further exacerbated by the fact that different studies of local ellipticals (arguably the likely present-day descendants of SMGs) have yielded a wide range of preferred IMFs including bottom-light (van Dokkum 2008), Kroupa (2001) (Gerhard et al. 2001; Cappellari et al. 2006), Salpeter (Grillo et al. 2009), and even bottom-heavy (van Dokkum \& Conroy 2010, 2011; see also Thomson \& Chary 2011).

Hence, since there is as yet no clear evidence that the IMF of SMGs is systematically different from that of other galaxies, in this paper we assume the Chabrier (2003) IMF (unless stated otherwise) to which all appropriate derived quantities are converted if necessary (values derived using the Salpeter 1955 IMF are divided by 1.8). This IMF has been derived for the Milky Way and represents an intermediate choice between the top-heavy and the bottom-heavy IMFs. We also note that this "Chabrier IMF" is essentially identical to the "Canonical IMF" recently summarised by Weidner et al. (2011). While we argue that this IMF is the natural choice on the basis of current evidence, it must still be accepted that we must live with a fundamental uncertainty of $\simeq \times 2$ in stellar masses until the uncertainty in the IMF in SMGs is resolved. However, we also note that the derived sSFR is unaffected by this IMF uncertainty, making it a particularly useful quantity for comparing the properties of different types of star-forming galaxies.

\subsection{Evolutionary models and star-formation histories}

It is well known that the choice of the evolutionary synthesis model used to fit the optical-infrared photometry can affect

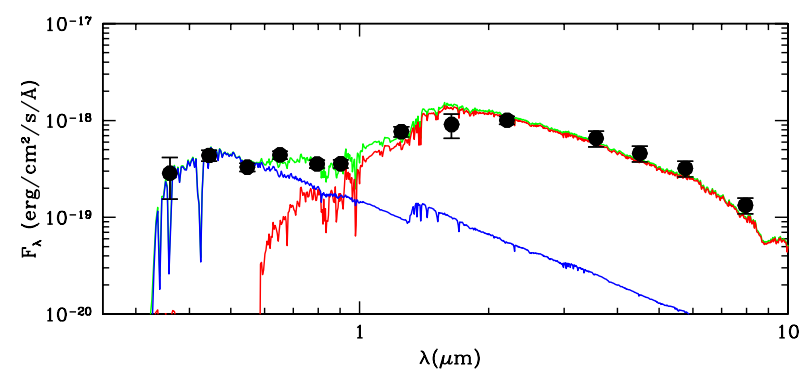

Fig. 1. Spectral energy distribution (flux density vs. observed wavelength) of SMMJ123606.85+621021.4 at the spectroscopic redshift $z=2.509$ fitted using the double-component star formation history method of Cirasuolo et al. (2010, C10). Black circles denote the data, red and blue curves are the SEDs of the old and young components, respectively, and the green curve is the sum of these two components. The older component dominates the near-IR light and the derived stellar mass, whereas the young component delivers almost all rest-frame ultraviolet flux density.

the derived stellar masses of any type of galaxy. The GRASIL spectral energy distribution (SED) templates (Silva et al. 1998; Iglesias-Páramo et al. 2007) used by M10 make use of the Padova stellar tracks (Alongi et al. 1993; Bressan et al. 1993; Bertelli et al. 1994; Fagotto et al. 1994a,b,c; Girardi et al. 1996, 2000). The input metallicity for GRASIL was calculated based on the chemical evolution model in which individual stellar populations are allowed to have metallicity between $0.2-5 Z_{\odot}$. The mean (median) metallicity derived for SMGs is $0.8 Z_{\odot}\left(0.6 Z_{\odot}\right)$.

H11 used Bruzual \& Charlot (2003) models (which are also based on the Padova library) and in addition explored the use of the Maraston (2005) models. H11 assumed solar metallicity in order to derive a typical mass-to-light ratio.

The stellar masses derived by H11 using Bruzual \& Charlot (2003) models are on average $\simeq 50 \%$ higher than those calculated using the Maraston (2005) models. This average difference is at least in part a consequence of the higher contribution of thermally pulsing asymptotic giant branch (TP-AGB) stars in the Maraston (2005) models, which reduces the derived massto-light ratio at rest-frame near-infrared wavelengths at stellar population ages $\simeq 1$ Gyr (a point discussed further below).

An apparently separate (but, it transpires, in fact connected) cause of differing stellar mass estimates is the chosen parameterisation of the star-formation histories (SFHs) of the galaxies in question. As noted by H11, due to their high redshift, and often rather red spectral-energy distributions, it is very difficult to recover robust SFHs for SMGs from photometric data (cf. Dye et al. 2008, 2010). This leads inevitably to difficulty in constraining galaxy ages and mass-to-light ratios.

In an attempt to account for this uncertainty, H11 explored the use of two alternative single-component SFHs, adopting either an instantaneous starburst or a continuous star-formation history. H11 then averaged the two resulting estimates to produce the adopted stellar mass for each galaxy. In contrast, M10 assumed a two-component SFH consisting of a smooth Schmidttype law $\left(S F R \propto M_{\text {gas }}\right)$ with a recent starburst superimposed commencing $50 \mathrm{Myr}$ before the observed galaxy SED was emitted, and with a mass in the range $0-9 \%$ of the infalling gas mass.

The assumption of a multi-component SFH generally naturally leads to higher mass-to-light ratios and, in turn, higher stellar masses than the use of a single-component model. This is because, while the starburst component can account for the ultraviolet (UV) emission (and the infrared emission from the UV-heated dust), the second (older) component is then free to 
M. J. Michałowski et al.: The stellar masses and specific star-formation rates of submillimetre galaxies

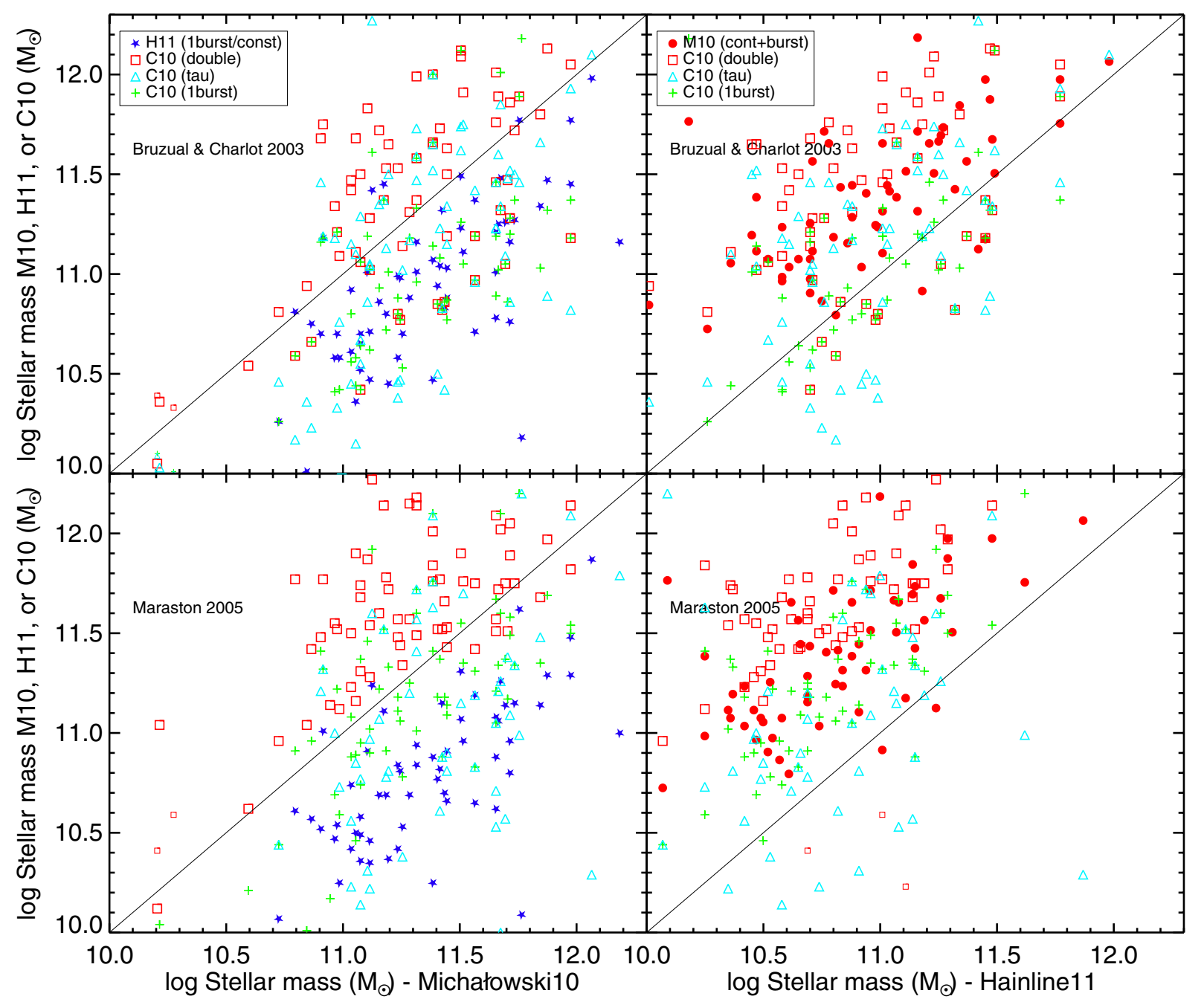

Fig. 2. Comparison of the stellar masses of SMGs as derived by M10, H11 and in the present work using the stellar population synthesis models of Bruzual \& Charlot (2003, top) and Maraston (2005, bottom). The different symbols indicate the stellar masses derived by M10 (red circles), H11 (blue stars), and our new estimates based on the SED modelling method of Cirasuolo et al. (2010, C10) for three alternative forms of star formation history: double component model (red squares), exponentially declining tau model (light blue triangles) and single burst (green pluses). All the derived masses have been converted to the Chabrier (2003) IMF. Small symbols denote $z<0.5$ SMGs. The M10 stellar masses are consistent with our new estimates for the double-component models, whereas the H11 stellar masses are consistent with single component models, and are on average a factor of $\simeq 2.5$ smaller than the double-component estimates (see Table 1 ).

contribute more stars with higher mass-to-light ratios (see Fig. 1 and also Schael et al., in prep.). By contrast, the use of a single instantaneous starburst model limits the age of the entire stellar population to the young age of the starburst required to match the UV emission (and thus the true stellar masses are inevitably under-estimated), while in the continuous star-formation model the current SFR is set by the UV emission and the total age is then limited in order not to overshoot the optical and the nearIR part of the spectrum (assuming the galaxy has always formed stars at the same high rate).

In order to further clarify the impact of the choice of stellar population synthesis models and star-formation histories on the derived stellar masses we have revisited the SED fitting of the Chapman et al. (2005) SMG sample. Specifically, we have used the method presented in Cirasuolo et al. (2007, 2010), based on the HyPERz package utilising both Bruzual \& Charlot (2003) and Maraston (2005) models and assuming three different forms of SFH: an instantaneous burst of star-formation, an exponentially declining star-formation rate (so-called tau models), and a double-component model composed of two instantaneous bursts with different ages and (independent) dust attenuations (an example is shown in Fig. 1 and all estimates are listed in Table A.1). In the double-component model the age of the young component was varied between $50 \mathrm{Myr}$ and $1.5 \mathrm{Gyr}$, and the old component was allowed to contribute $0-100 \%$ of the nearIR emission while its age was varied over the range $1-6 \mathrm{Gyr}$. Solar metallicity was assumed. These models were run using the Chabrier IMF.

The results of this modelling are illustrated in Fig. 2 and summarised in Table 1, where the mean and median values of $M_{*}$ and SSFR (as well as ages and contributions of the older population) for the SMGs are tabulated for the full range of different stellar population synthesis models and star-formation histories explored in M10, H11 and the present work (it is not possible to distinguish the models based on the goodness of fit). What is immediately evident from this table is that the results are strongly dependent on the assumed parameterisation of the star-formation history (see also Bussmann et al. 2012), as is the impact of assuming different population synthesis models. In particular, it is noteworthy that the Maraston (2005) models only result in 
Table 1. Comparison of stellar masses and specific star formation rates of SMGs calculated using various methods, stellar population models and star formation histories.

\begin{tabular}{|c|c|c|c|c|c|c|c|c|c|}
\hline $\mathrm{Fit}^{a}$ & $\mathrm{SSP}^{b}$ & $\mathrm{SFH}^{c}$ & $\begin{array}{c}\text { Mean } M_{*} \\
\left(\log M_{\odot}\right)\end{array}$ & $\begin{array}{c}\text { Median } M_{*} \\
\left(\log M_{\odot}\right) \\
\end{array}$ & $\begin{array}{c}\text { Mean SSFR } \\
\left(\mathrm{Gyr}^{-1}\right)\end{array}$ & $\begin{array}{c}\text { Median SSFR } \\
\left(\mathrm{Gyr}^{-1}\right)\end{array}$ & $\begin{array}{c}\operatorname{Age}_{Y}{ }^{d} \\
(\mathrm{Gyr})\end{array}$ & $\begin{array}{c}\operatorname{Age}_{\mathrm{O}}{ }^{e} \\
(\mathrm{Gyr})\end{array}$ & $\operatorname{Frac}_{\mathrm{O}}{ }^{f}$ \\
\hline M10 & Padova & cont+burst & $11.32 \pm 0.05$ & $11.31_{-0.06}^{+0.07}$ & $2.86 \pm 0.43$ & $1.74_{-0.46}^{+0.17}$ & 0.050 & 1.856 & 0.84 \\
\hline H11 & $\mathrm{BC} 03$ & 1burst/const & $10.87 \pm 0.06$ & $10.88_{-0.07}^{+0.11}$ & $9.71 \pm 1.99$ & $4.60_{-0.92}^{+1.22}$ & 0.820 & $\ldots$ & $\cdots$ \\
\hline H11 & M05 & 1burst/const & $10.71 \pm 0.06$ & $10.74_{-0.05}^{+0.08}$ & $12.96 \pm 2.17$ & $6.85_{-2.09}^{+1.56}$ & 0.860 & $\ldots$ & $\ldots$ \\
\hline $\mathrm{C} 10$ & $\mathrm{BC} 03$ & double & $11.44 \pm 0.08$ & $11.47_{-0.10}^{+0.11}$ & $10.87 \pm 8.68$ & $1.35_{-0.31}^{+0.21}$ & 0.191 & 1.332 & 0.87 \\
\hline $\mathrm{C} 10$ & ВC03 & tau & $11.16 \pm 0.10$ & $11.18_{-0.05}^{+0.11}$ & $17.36 \pm 11.98$ & $2.56_{-0.71}^{+1.00}$ & 1.178 & $\ldots$ & $\ldots$ \\
\hline $\mathrm{C} 10$ & ВC03 & 1 burst & $11.06 \pm 0.10$ & $11.02_{-0.06}^{+0.06}$ & $14.88 \pm 8.69$ & $3.07_{-0.47}^{+1.16}$ & 0.246 & $\ldots$ & $\ldots$ \\
\hline $\mathrm{C} 10$ & M05 & double & $11.73 \pm 0.06$ & $11.72_{-0.12}^{+0.03}$ & $1.25 \pm 0.19$ & $0.67_{-0.11}^{+0.25}$ & 0.075 & 1.777 & 0.66 \\
\hline $\mathrm{C} 10$ & M05 & tau & $10.46 \pm 0.13$ & $10.78_{-0.17}^{+0.07}$ & $167.34 \pm 47.42$ & $5.16_{-0.78}^{+6.38}$ & 0.767 & $\ldots$ & $\ldots$ \\
\hline $\mathrm{C} 10$ & M05 & 1burst & $11.24 \pm 0.07$ & $11.22_{-0.04}^{+0.12}$ & $8.43 \pm 4.57$ & $1.90_{-0.43}^{+0.49}$ & 0.198 & $\cdots$ & $\cdots$ \\
\hline
\end{tabular}

Notes. The Chabrier (2003) IMF is assumed. Errors are standard deviations for the means and bootstrap 68\% ranges for medians. ${ }^{(a)}$ SED fitting method presented in M10 (Michałowski et al. 2010a), H11 (Hainline et al. 2011), or C10 (Cirasuolo et al. 2010). ${ }^{(b)}$ Single stellar population models being either Padova tracks, BC03 (Bruzual \& Charlot 2003) or M05 (Maraston 2005). ${ }^{(c)}$ Assumed star formation history being either a continous SFH with a burst (cont+burst), an average of the single burst and constant (1 burst/const), double burst (double), single burst (1 burst), or exponentially declining (tau). ${ }^{(d)}$ Mean age of the younger stellar component. ${ }^{(e)}$ Mean age of the older stellar component. ${ }^{(f)}$ Mean fractional contribution to the total stellar mass of the older component.

significantly lower stellar masses (and hence substantially higher sSFRs) than the Bruzual \& Charlot (2003) models when a singlecomponent exponentially-declining tau-model SFH is assumed. We investigated the origin of this effect, and found that, when allowed only single-component tau models, the mean preferred age of the Maraston (2005) model fits is $\simeq 0.8$ Gyr (see Table 1), which corresponds closely to the time at which the enhanced TP-AGB star contribution in the Maraston (2005) models has maximum impact. This result provides further evidence that the combination of tau SFHs and the Maraston (2005) models is not appropriate for SMGs.

Reassuringly, however, there is no significant difference between the masses derived with these two alternative models for a single burst SFH. This is because, in order to reproduce the star-forming nature of SMGs, the single burst fitting typically results in much younger ages of $\simeq 0.2 \mathrm{Gyr}$, corresponding to the time when the TP-AGB contribution is small and does not have a significant influence on the derived stellar masses.

As expected (see above discussion of the M10 modelling approach), the stellar masses obtained using the double component SFH are systematically larger. Especially, the Maraston (2005) models with the double SFH yield stellar masses higher by $\simeq 1$ dex than these derived assuming the tau SFHs with these models.

In summary, the derived stellar masses depend primarily on the assumed parameterisation of the SFH, and the different evolutionary synthesis models only yield significantly different results if the single-component exponentially-declining SFH is adopted (due primarily to the extreme behaviour of the Maraston 2005 , models with this adopted parameterization of SFH). Since, as we argue below, a model of a rapidly starforming galaxy in which the SFR is assumed to have been even higher at all previous epochs is obviously physically unrealistic for SMGs, the preferred choice of evolutionary synthesis model is actually of less importance in deriving the stellar masses of SMGs than might have been supposed. Nevertheless, in the next section, we do, for completeness, review the arguments for and against the choice of the Maraston (2005) or Bruzual \& Charlot (2003) models.

Of more significance is the fact that, as can be seen from Table 1, our new two-component models using the
Bruzual \& Charlot (2003) models yield a value very similar to that derived by M10 using a different form of two-component model, and a different evolutionary synthesis model (albeit based on the same stellar tracks as the Bruzual \& Charlot 2003 models). Thus, consistent average stellar masses are produced by models which allow recent and past SFR to be decoupled, regardless of whether the earlier star-formation is parametrised as a single burst or a more extended period of star formation. This consistency is both reassuring and helpful given that, as we argue further below, a SFH history allowing potentially decoupled recent and past star-formation activity is rather obviously appropriate for objects selected on the basis of recent very violent star formation within an already metal enriched environment.

\subsection{Choice of evolutionary synthesis model}

Recent years have seen some vigorous debate over whether the stronger TP-AGB contribution introduced into evolutionary synthesis models by Maraston (2005) is justified by the data.

Some workers have argued for the superiority of the Maraston (2005) models based on derived galaxy stellar masses (van der Wel et al. 2006a,b), on derived photometric redshifts (Maraston et al. 2006), on average broad-band SEDs (Maraston et al. 2006; Cimatti et al. 2008), on optical and near-infrared colours (MacArthur et al. 2010), and by comparison with semianalytical models (Henriques et al. 2011). Moreover, Zibetti et al. (2009) have claimed that stellar mass determinations based on $i$ - and $H$-band luminosities are more consistent with each other when long-lived TP-AGB stars are introduced.

However Eminian et al. (2008) showed that Maraston (2005) models predict excessively red optical colours, and recently Kriek et al. (2010) have found that the average spectral energy distribution of post-starburst galaxies at $0.7 \lesssim z \lesssim 2.0$ does not appear to display the excess near-infrared flux predicted from the TP-AGB contribution in the Maraston (2005) models. Indeed, they demonstrate that the Bruzual \& Charlot (2003) model provides a much better description of the the data, even at the age when the TP-AGB contribution should be near maximum. In addition, Conroy \& Gunn (2010, their Figs. 2 and 16) have found that the Maraston (2005) models predict excessively red colours 
for post-starburst galaxies and for star clusters in the Magellanic Clouds. Finally, Zibetti et al. (2012) did not find any strong carbon feature of TP-AGB stars in the near-IR spectra of poststarburst galaxies.

The work presented here cannot be used to definitively resolve this issue, but we note that the extreme outlying nature of the tau-model Maraston (2005) results shown in Table 1 arguably provides some further evidence against the plausibility and appropriateness of the strong TP-AGB contribution adopted in these models for tau SFHs. Moreover, we note that the Maraston (2005) models with the double SFH yield the highest median stellar masses for SMGs.

We also note that, confining the comparison to the use of the Chabrier (2003) IMF and the Bruzual \& Charlot (2003) models, the median stellar mass of SMGs derived by M10 $\left(M_{*}=2 \times\right.$ $\left.10^{11} M_{\odot}\right)$ is in fact only a factor of $\simeq 2.5$ higher than that reported by $\mathrm{H} 11\left(M_{*}=7.6 \times 10^{10} M_{\odot}\right)$.

\subsection{Choice of star-formation history}

The key issue for stellar mass determination, therefore, appears to be the choice of how to parameterize the SFH of SMGs. Reassuringly, however, the results do not appear to be very sensitive to precisely how this is parameterized, provided the SFH allows past and "present" star-formation activity to be, at least in principle, disconnected. This can be seen from the fact that the different two-component models based on the Padova tracks listed in Table 1 yield average stellar masses in the range $M_{*}=2-3 \times 10^{11} M_{\odot}$.

It only therefore remains to consider if these two-component models should be preferred to single-component burst or tau models. In fact, at least for SMGs, this is a relatively straightforward argument, since both the single-burst and tau models both enforce the condition that the star-formation rates in SMGs were always higher at epochs earlier than that at which the object has been selected due to its extreme star-formation rate. Indeed this problem has already been discussed by several authors including Maraston et al. (2010). They concluded that, for high-redshift galaxies, the assumption of an exponentially decreasing SFH (as well as constant SFH to some extent) leads to unrealistically low ages and under-estimated stellar masses, because the SED fit is completely dominated by young stars. In fact Maraston et al. (2010) found an exponentially increasing SFH could provide a much better description of data. Such SFHs are conceptually similar to those used in M10, with the SFR being highest close to the epoch at which the observed galaxy SED was emitted, and indeed there is growing evidence in favour of rising SFHs in the wider population of galaxies studied at high redshift (e.g. Finlator et al. 2011; Papovich et al. 2011).

The lack of either two-component models or rising SFHs in the analysis of H11 (or indeed Wardlow et al. 2011) can therefore explain all of the remaining discrepancy in the average stellar masses reported by M10 and H11 since, as can be seen from Table 1, the use of a double component SFH results in stellar masses of SMGs being $\simeq 3$ times larger than that deduced using the single burst model and $\simeq 2$ times larger than that derived using the tau models. This is also illustrated in Fig. 2, where the masses derived assuming single-component SFHs (light blue triangles and green plusses) are systematically lower than those derived assuming the more realistic double-component SFHs (red squares). This conclusion is consistent with the findings of Schael et al. (in prep. $)^{1}$ who found that the stellar masses of

\footnotetext{
1 See also Schael (2009).
}

the SHADES SMGs (Coppin et al. 2006) derived using a twocomponent $\mathrm{SFH}$ are on average a factor of $\simeq 2-3$ higher than when derived using a single-component SFH.

\subsection{Other model-fitting issues}

\subsubsection{Wavelength range}

The wavelength range used in the SED model fitting could potentially affect the resulting derived stellar masses. For completeness we therefore tested whether the difference in the SMG stellar mass estimates derived by M10 and $\mathrm{H} 11$ is not, at least in part, due to the fact that M10 fitted the entire multi-frequency dataset up to radio wavelengths, whereas $\mathrm{H} 11$ confined attention to observed $\lambda \leq 8.0 \mu \mathrm{m}$. To test this we simply repeated the analysis performed by M10, but this time restricting the model fitting to photometry at optical, near-infrared and IRAC wavelengths. We obtained almost identical results with a median stellar mass of $\simeq 1.7 \times 10^{11} M_{\odot}$ instead of $\simeq 1.9 \times 10^{11} M_{\odot}$, and hence conclude that the longer-wavelength data have no significant influence on the derived stellar masses. This is reassuring and is easily explained since the emission at far-infrared wavelengths depends only on the properties of the dust and of the younger stellar population that is heating the dust, and should be essentially unaffected by the older stars which dominate the total stellar masses of SMGs (M10).

\subsubsection{AGN contamination}

SMGs are believed to suffer only a minor $(\simeq 10-30 \%)$ contamination of their bolometric luminosities by active galactic nuclei (AGNs; Alexander et al. 2005, 2008; Menéndez-Delmestre et al. 2007, 2009; Valiante et al. 2007; Pope et al. 2008; Hainline et al. 2009; Murphy et al. 2009; Watabe et al. 2009, M10). Here we consider whether the presence of AGNs in SMGs can significantly influence the derivation of their typical stellar masses.

$\mathrm{H} 11$ reported that, for $\simeq 50 \%$ of SMGs, non-stellar (AGN) power-law features contribute $<10 \%$ in the rest-frame $H$-band, whereas, for $\simeq 10 \%$ of SMGs, the contribution is $>50 \%$. However, even for those objects which do appear to have a significant AGN contribution at observed IRAC wavelengths, it is not obvious that the stellar masses based on our full multi-wavelength fitting will be significantly distorted, especially given the typically large $(\simeq 20 \%)$ photometric errors generally assigned to IRAC data in the SED fitting process. In fact we find that the stellar masses reported by $\mathrm{H} 11$ are consistent with our estimates for single component models (Fig. 2 and Table 1), without making any corrections for possible AGN contributions. We therefore do not find any evidence to support the idea that the lower stellar masses reported by $\mathrm{H} 11$ are due to the subtraction of an AGN component. Rather, as described above, it seems clear that the lower stellar masses deduced by H11 arise, primarily, from the adoption of a single-component SFH.

We also note that the presence of any power-law features in the IRAC data should not have strongly affected the mass estimates of M10, simply because the SED fits were not forced to reproduce the power-law features and, in fact, the best-fitting stellar models tend to under-estimate the longer wavelength IRAC data when the power-law component appears to be present (see Fig. A1 of M10). Moreover, we do not find any correlation between the power-law fraction given by $\mathrm{H} 11$ and the stellar masses or $M_{*} / L_{K}$ derived by M10, which would be expected if the power-law features were biasing the M10 stellar mass estimates high. 
Table 2. Stellar, gas and dynamical masses of SMGs (in units of $10^{11} M_{\odot}$ ).

\begin{tabular}{lcccccc}
\hline \hline SMG & $M_{*(\mathrm{M} 10)}{ }^{a}$ & $M_{*(\mathrm{C} 10)}{ }^{b}$ & $M_{*(\mathrm{H} 11)}^{c}$ & $M_{\mathrm{gas}}$ & $M_{\mathrm{dyn}}^{\text {highCO } d}$ & $M_{\mathrm{dyn}}^{\mathrm{CO}(1-0) e}$ \\
\hline SMMJ123549.44+621536.8 & $2.66_{-1.33}^{+2.65}$ & $0.66_{-0.33}^{+0.66}$ & $2.09_{-0.47}^{+0.60}$ & $2.07 \pm 1.73^{f}$ & $0.96 \pm 0.44^{g}$ & $2.30 \pm 0.40^{f}$ \\
SMMJ123618.33+621550.5 & $2.78_{-1.39}^{+2.77}$ & $4.27_{-2.13}^{+4.25}$ & $0.76_{-0.08}^{+0.09}$ & $0.35 \pm 0.11^{g}$ & $2.18 \pm 0.18^{g}$ & $4.36 \pm 0.36^{i}$ \\
SMMJ123634.51+621241.0 & $2.54_{-1.27}^{+2.53}$ & $0.71_{-0.35}^{+0.70}$ & $0.87_{-0.11}^{+0.13}$ & $0.25 \pm 0.08^{g}$ & $1.12 \pm 0.25^{g}$ & $2.24 \pm 0.51^{i}$ \\
SMMJ123707.21+621408.1 & $4.95_{-2.47}^{+4.93}$ & $1.12_{-0.56}^{+1.12}$ & $1.82_{-0.27}^{+0.32}$ & $1.69 \pm 1.41^{f}$ & $2.78 \pm 1.05^{g}$ & $2.20 \pm 0.60^{f}$ \\
SMMJ131201.17+424208.1 & $1.27_{-0.63}^{+1.27}$ & $6.76_{-3.37}^{+6.73}$ & $1.02_{-0.25}^{+0.33}$ & $1.34 \pm 0.50^{g}$ & $9.50 \pm 2.38^{g}$ & $19.01 \pm 4.76^{i}$ \\
SMMJ163650.43+405734.5 & $2.06_{-1.03}^{+2.05}$ & $3.80_{-1.90}^{+3.78}$ & $1.45_{-0.32}^{+0.42}$ & $2.56 \pm 2.14^{f}$ & $3.50 \pm 1.79^{g}$ & $4.10 \pm 0.80^{f}$ \\
SMMJ163658.19+410523.8 & $3.27_{-1.63}^{+3.26}$ & $8.13_{-4.05}^{+8.09}$ & $1.29_{-0.26}^{+0.33}$ & $2.95 \pm 2.46^{f}$ & $1.40 \pm 0.88^{g}$ & $5.80 \pm 0.40^{f}$ \\
SMMJ163706.51+405313.8 & $4.52_{-2.25}^{+4.49}$ & $10.23_{-5.10}^{+10.18}$ & $1.62_{-0.21}^{+0.24}$ & $0.24 \pm 0.07^{h}$ & $3.40^{h}$ & $6.80^{i}$ \\
SMMJ221735.15+001537.2 & $1.30_{-0.65}^{+1.30}$ & $1.91_{-0.95}^{+1.90}$ & $0.51_{-0.06}^{+0.06}$ & $0.30 \pm 0.07^{h}$ & $2.80^{h}$ & $5.60^{i}$ \\
\hline
\end{tabular}

Notes. ${ }^{(a)}$ Michałowski et al. (2010a) with Chabrier (2003) IMF. ${ }^{(b)}$ From this work using the method of C10 for Bruzual \& Charlot (2003) models and double SFH. ${ }^{(c)}$ Hainline et al. (2011) for Bruzual \& Charlot (2003) models. ${ }^{(d)}$ Dynamical mass from high exitation CO line. ${ }^{(e)}$ Dynamical mass from $\mathrm{CO}(1-0)$ line. ${ }^{(f)}$ Ivison et al. (2011). ${ }^{(g)}$ Engel et al. (2010). ${ }^{(h)}$ Greve et al. (2005). ${ }^{(i)}$ Obtained by multiplying the $M_{\text {dyn }}^{\text {highco }}$ by a factor of 2, the average correction from high excitation lines to $\mathrm{CO}(1-0)$ derived for four SMGs with $\mathrm{CO}(1-0)$ data. Mass errors for M10 and C10 are simply a factor of 2 .

\subsubsection{Lensing}

If SMGs are lensed then the stellar masses derived by M10 and H11 should be corrected down. At least some of the brightest SMGs are thought to be lensed (Ivison et al. 1998; Downes \& Solomon 2003; Dunlop et al. 2004; Kneib et al. 2004; Motohara et al. 2005; Wilson et al. 2008; Berciano Alba et al. 2010; Knudsen et al. 2010; Negrello et al. 2010; Swinbank et al. 2010b; Vieira et al. 2010; Tamura et al. 2010; Hezaveh \& Holder 2011; Ikarashi et al. 2011). However, Lima et al. (2010, Fig. 2) found that the number counts of SMGs with $S_{850}<20 \mathrm{mJy}$ do not require any lensing correction. Moreover, the modelling of Paciga et al. (2009, Fig. 6) showed that only $\lesssim 10 \%$ of SMGs with $S_{850}<15 \mathrm{mJy}$ at $z<3$ are magnified by a factor of $>2$. Hence, the lensing correction for SMGs in the $850 \mu \mathrm{m}$ flux-density range considered here is likely to be small on average.

\section{Consistency tests for stellar masses}

\subsection{Dynamical masses}

The estimates of the dynamical and $\mathrm{H}_{2}$ gas masses from $\mathrm{CO}$ lines detected for SMGs provide a consistency check on the stellar mass estimates. Specifically, assuming that the extent of the COemitting gas is the same as the extent of the stellar component, the sum of the gas and stellar masses has to be less than the dynamical mass. Using this approach Engel et al. (2010) derived a mean ratio $\left(M_{*}+M_{\text {gas }}\right) / M_{\text {dyn }} \simeq 2.1$ for seven SMGs using the stellar masses of M10 (corrected to the Chabrier 2003 IMF) and concluded that the stellar masses derived by M10 are inconsistent with their gas and dynamical masses. As explained below, we do not find that this is the case.

There is, of course, some uncertainty and controversy over the calculation of dynamical and molecular gas masses from CO line observations (e.g. Genzel et al. 2003; Ivison et al. 2011). Perhaps of most relevance for the present discussion is the fact that Engel et al. (2010) used high excitation lines (CO $J=7-6$, $6-5,4-3$ and 3-2), which trace denser and hotter gas, very likely confined to the more central regions of a galaxy. Therefore, the derived dynamical masses could easily refer to regions smaller than the extent of the stellar component, making it difficult to compare them directly with the stellar masses. Indeed, based on low excitation $\mathrm{CO}(1-0)$ lines Ivison et al. (2011) found dynamical masses higher by a factor $\simeq 2.4-4.1$ for two out of three SMGs, for which Engel et al. (2010) claimed a significant discrepancy between stellar and dynamical mass (HDF 76 and N2 850.2; the third one was not observed by Ivison et al.). Moreover, recently Riechers et al. (2011) found CO(1-0) sizes $\simeq 60-90 \%$ larger than that reported by Engel et al. (2010) for two additional SMGs.

Interestingly, the mean $\mathrm{CO}$ half-light radius obtained by Engel et al. (2010) of $2.4 \mathrm{kpc}$ is indeed $15-40 \%$ smaller than the typical half-light radius of the near-infrared emission dominated by the stellar component $(2.8-3.4 \mathrm{kpc}$; Swinbank et al. 2010a; Mosleh et al. 2011; Targett et al. 2011). Moreover, one out of three SMGs for which Engel et al. (2010) claimed inconsistency with the M10 stellar mass (N2 850.2) was observed by Swinbank et al. (2010a) who measured the stellar extent to be $\simeq 3$ times larger than the extent of the $\mathrm{CO}(7-6)$ found by Engel et al. (2010). This reinforces the likelihood that the dynamical masses of the problematic SMGs have been under-estimated, as the stellar component is more extended than the high-excitation gas.

This argument is supported by hydrodynamical simulations of SMGs (Narayanan et al. 2009, 2010b; Hayward et al. $2011 \mathrm{a}, \mathrm{b}$ ), where only $\simeq 30 \%$ of the stellar mass is distributed over the inner radius of $2.5 \mathrm{kpc}$, roughly the size over which Engel et al. (2010) calculated dynamical masses. This correction to the dynamical masses would be enough to remove the inconsistency with the stellar masses of M10, even though some simulations predict that the usual conversions result in the dynamical masses overpredicted by a factor of $\simeq 1.5-1.9$ (Narayanan et al. 2009, Fig. 10 and Eq. (6)).

In an attempt to overcome these systematics, and facilitate a fairer comparison of dynamical and stellar masses, we focus here on the newly available $\mathrm{CO}(1-0)$ dynamical masses which, as described above, appear to better trace the full extent of the SMGs, yielding somewhat larger sizes and masses. Stellar, gas and dynamical masses are available for 9 of the galaxies in our sample, including two CO detections made by Greve et al. (2005). These data ${ }^{2}$ are presented in Table 2.

In the final column of this table we give the CO (1-0) dynamical mass as our best estimate of the dynamical mass of

\footnotetext{
${ }^{2}$ Note that Engel et al. (2010) used the stellar masses reported initially in Hainline (2008) whereas we quote the corrected values from H11.
} 
M. J. Michałowski et al.: The stellar masses and specific star-formation rates of submillimetre galaxies

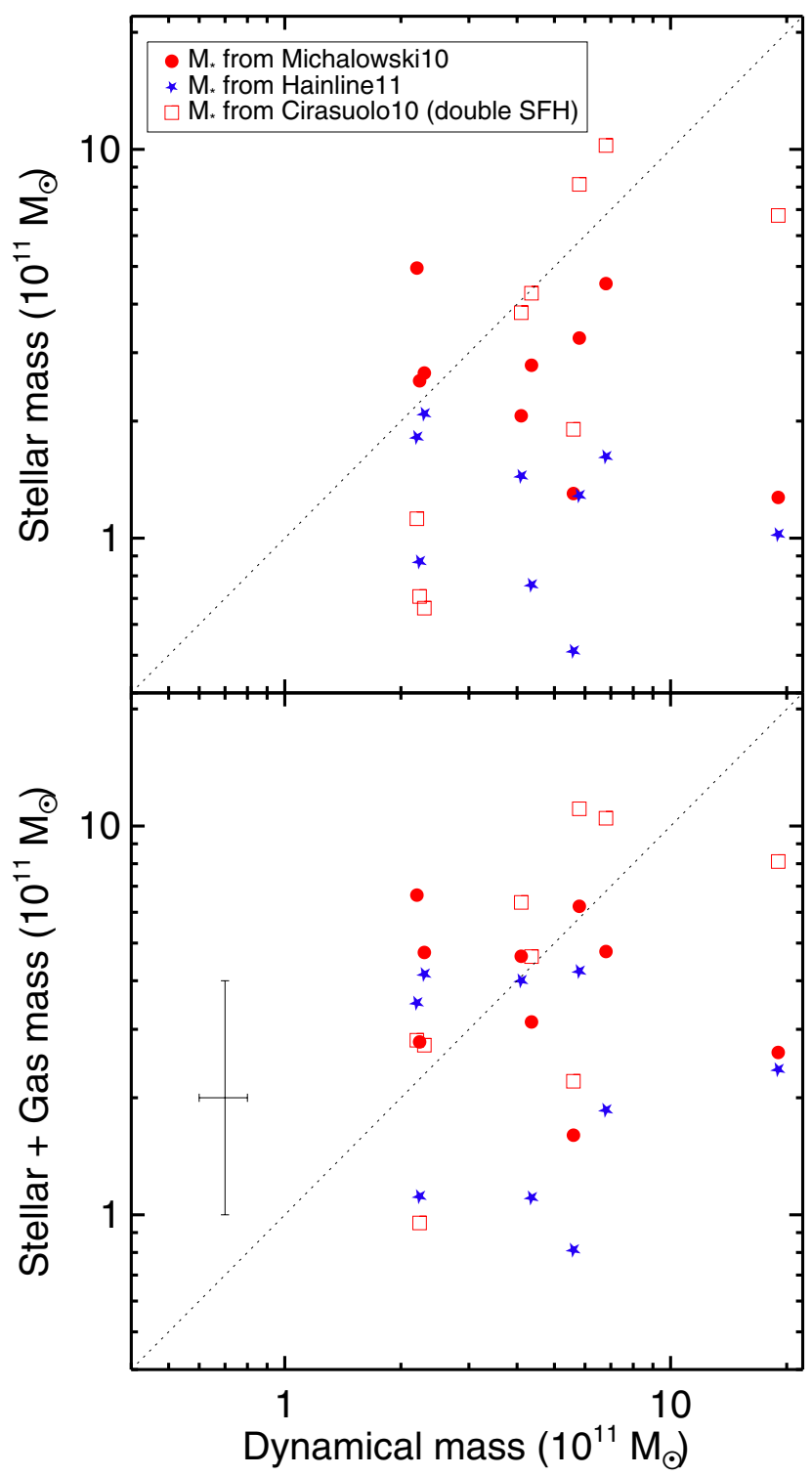

Fig. 3. Stellar mass $\left(M_{*}\right)$ versus dynamical mass $\left(M_{\mathrm{dyn}}\right)$ estimated from $\mathrm{CO}(1-0)$ (upper panel), and stellar $+\mathrm{H}_{2}$ gas mass $\left(M_{*}+M_{\mathrm{gas}}\right)$ versus $M_{\mathrm{dyn}}$ (lower panel) for the $9 \mathrm{SMGs}$ for which the necessary results have been compiled in Table 2 . The data points derived from the stellar mass estimates of $\mathrm{H} 11$ are indicated by blue stars, those based on the stellar mass estimates of M10 are indicated by red circles, while the new double-component $\mathrm{C} 10$ values are indicated by red squares. In principle, only the area below the dotted line represents a consistent set of galaxy properties with $M_{\text {gas }}+M_{*} \leq M_{\text {dyn }}$, but because of the typical scatter (indicated by the error bars in the lower panel) and unknown inclinations, $\simeq 2-3$ out of the 9 individual SMGs are in fact expected to exceed this apparent threshold (see Sect. 3.1 for details). We see that, contrary to the conclusion of Engel et al. (2010), there is no evidence that the stellar masses derived by M10 using the Chabrier IMF are in conflict with the dynamical mass estimates, even allowing for estimates of the additional gas mass. Indeed, the average values of these ratios favour the M10 stellar mass estimates as the most consistent with the available data (see Sect. 3.1).

each galaxy. Where available (4 SMGs) this number has been taken directly from the CO (1-0) observations performed by Ivison et al. (2011), while for the remaining objects this number has been inferred from the higher-order $\mathrm{CO}$-line dynamical masses by multiplying them by the average correction factor for the aforementioned 4 SMGs for which the dynamical masses of Ivison et al. (2011) and Engel et al. (2010) can be directly compared.

In Fig. 3 we then plot, for these 9 SMGs, $M_{*}$ and also $M_{\text {gas }}+$ $M_{*}$ as a function of $M_{\mathrm{dyn}}$, with the dynamical masses taken from the final column in Table 2, and the stellar mass estimates from $\mathrm{H} 11, \mathrm{M} 10$ and the present work (double-component C10 model) indicated by the different symbols.

From Fig. 3 it can be seen that, within the errors, there is no evidence that even the higher stellar mass determinations for any object exceed the estimated dynamical mass. At first sight it might seem that there could be more of a problem (for all mass determinations) for $M_{*}+M_{\mathrm{gas}}$, but this is not really the case, both because of uncertainty in $M_{\text {gas }}$, and because of the potential effects of varying inclination angle on $M_{\text {dyn }}$. Specifically, while the unknown inclination $(i)$ of gas discs should not influence the average $\left(M_{\mathrm{gas}}+M_{*}\right) / M_{\mathrm{dyn}}$ ratio, if the real inclination of an individual galaxy is lower than the assumed average, then its derived $M_{\text {dyn }}$ is inevitably too low, which can make it appear to be inconsistent with the (orientation-independent) derived stellar mass. To quantify this effect we considered 100000 galaxies with real $\left(M_{\text {gas }}+M_{*}\right) / M_{\text {dyn }}=1$ (or 0.5$)$ with inclinations distributed uniformly in three dimensions, i.e. with a mean $\left\langle\sin ^{2} i\right\rangle=2 / 3$. We found that the adoption of this mean value for all galaxies results in $\simeq 18 \%$ (9\%) of them appearing to exceed the allowed $\left(M_{\text {gas }}+M_{*}\right) / M_{\text {dyn }}=1$ by a factor of $>2$ and $\simeq 12 \%(7 \%)$ by a factor of $>3$. Hence, in a sample of $\simeq 10$ galaxies with random unknown inclinations, we would expect 1-2 of them to significantly exceed the allowed ratio, simply because of the unknown inclination of any molecular disc in an individual object.

In summary, one should expect, given the effects of random inclination and uncertainty in the measurement of $M_{*}, M_{\mathrm{gas}}$ and $M_{\text {dyn }}$, that a few galaxies should lie above the one-to-one relation indicated by the dotted line in Fig. 3, and it is the mean (or median) value of the mass ratios which need to be considered when reviewing the viability of the stellar masses. Working from the numbers given in Table 2 , for the ratio $M_{*} / M_{\text {dyn }}$ the mean (median) values are $0.36(0.24)$ for the $\mathrm{H} 11$ stellar masses, 0.80 (0.64) for M10, and 0.94 (0.78) for the new double-component C10 values. Thus, adoption of the lower H11 stellar masses would imply that only $\simeq 1 / 3$ of the dynamical mass of a typical SMG can be accounted for by $M_{*}$. In contrast, the M10 and C10 results would imply that $M_{*}$ for a typical SMG is $\simeq 3 / 4 \times M_{\text {dyn }}$. If the values of $M_{\text {gas }}$ in Table 2 are taken at face value then this latter scenario would appear to be more consistent with the data, as for the ratio $\left(M_{*}+M_{\mathrm{gas}}\right) / M_{\mathrm{dyn}}$ the mean (median) values are $0.71(0.50)$ for the H11 stellar masses, 1.15 (1.07) for M10, and 1.09 (1.19) for the new double-component $\mathrm{C} 10$ values. This then provides further evidence that the stellar mass estimates of H11 are a little too low, the new $\mathrm{C} 10$ double-component values are perhaps a little high, and the M10 results appear to provide the best description of the data.

We conclude that the latest estimates of the dynamical masses of SMGs are in very good accord with the stellar masses of SMGs estimated by M10 with the Chabrier (2003) IMF.

\subsection{Number density of massive galaxies}

Another consistency check on the plausibility of the stellar-mass estimates is to assess how the number density of SMGs compares with the number density of galaxies in general in the same implied stellar-mass range, at both lower and comparable redshifts. 
Drory et al. (2004) reported the cumulative number density of galaxies $n\left(M_{*}>1.1 \times 10^{11} M_{\odot}\right)=3.16-0.64 \times 10^{-4} \mathrm{Mpc}^{-3}$ at redshifts $z=0.5-1.1$ (their Fig. 6 and Table 3, after conversion of their mass limit to the Chabrier 2003 IMF). Restricting the analysis to the SMGs at $z>1.4$, and using the comoving volumes given by M10 we obtain $n_{\mathrm{SMG}}\left(M_{*}>1.1 \times 10^{11} M_{\odot}\right)=$ $7.0 \times 10^{-6} \mathrm{Mpc}^{-3}$ for the $M_{*}$ values of M10 and $3.8 \times 10^{-6} \mathrm{Mpc}^{-3}$ for the $M_{*}$ values of H11. Hence, even for the M10 stellar mass estimates the number density of SMGs is $\simeq 30$ times lower than that of massive galaxies at lower redshifts and therefore the number of massive SMGs is clearly not inconsistent with the number density of massive galaxies at lower redshifts, even allowing for a relatively modest and plausible duty cycle (see below). In addition, from the mass comparisons discussed in the previous subsection, further conversion of the observed molecular gas reservoir into stars, even if extremely efficient, can at most increase the measured M10 stellar masses by $\simeq 30-50 \%$ (or, equivalently, the $\mathrm{H} 11$ masses by $\simeq 100 \%$ ), and so this basic conclusion is not seriously affected by potential further star formation.

The redshift range $1.4<z<3.6$ corresponds to a time interval of 2.7 Gyr. Given the typical gas reservoirs of $M_{\text {gas }} \simeq$ $1 \times 10^{11} M_{\odot}$ listed in Table 2 it can be seen that the typical gas exhaustion timescale is $\simeq 200 \mathrm{Myr}$ (assuming a typical $S F R$ of $\simeq 500 M_{\odot} \mathrm{yr}^{-1}$ ). If one further assumes that a typical SMG is observed mid-way through its final violent star-forming peak, then a reasonable estimate of the submillimetre luminous lifetime of an SMG is $400 \mathrm{Myr}$ (see also Bouché et al. 2005). This implies a duty-cycle number density correction factor of $\simeq 6-7$ for the redshift interval in question. If one further factors an incompleteness correction of a factor of $\simeq 3.5$ as derived by M10, then the implied true number density of potential SMGs in this era is close to, but still less than the best estimate of $M_{*}>1.1 \times 10^{11} M_{\odot}$ galaxies at $z \simeq 1.1$. Hence, the conclusion that the stellar masses of SMGs are typically $M_{*} \simeq 2 \times 10^{11} M_{\odot}$ appears to be perfectly consistent with the number density of massive galaxies at $z \simeq 1$.

A similar conclusion can be reached comparing to the number density reported by Pozzetti et al. (2007, Fig. 11) and Kajisawa et al. (2009, Fig. 16): $n\left(M_{*}>6 \times 10^{10} M_{\odot}\right) \simeq 10^{-3}-$ $2 \times 10^{-4} \mathrm{Mpc}^{-3}$ at $z=0-2$, whereas the corresponding values for SMGs are $n_{\mathrm{SMG}}\left(M_{*}>6 \times 10^{10} M_{\odot}\right)=8.7 \times 10^{-6}$ and $5.6 \times 10^{-6} \mathrm{Mpc}^{-3}$.

Finally, we integrated the mass function at $z=3-3.5$ given by Caputi et al. (2011) for $M_{*}>10^{11} M_{\odot}$ galaxies, obtaining $7 \times 10^{-6} \mathrm{Mpc}^{-3}$. This rather narrow redshift interval corresponds to an elapsed time of only $350 \mathrm{Myr}$, and so, following the above arguments, the duty cycle correction should be $\simeq$ unity. It is therefore perhaps unsurprising that this number density is rather similar to the number density of SMGs.

\subsection{Numerical simulations of SMGs}

Figure 4 shows submillimetre flux density versus stellar mass for a set of idealized hydrodynamic simulations of merging and isolated disk galaxies, where 3-D radiative transfer has been performed to calculate the submillimetre flux. The simulations are similar to those described in Narayanan et al. (2009, 2010a,b); see Hayward et al. (2011a,b) for a description of the differences. Note that the plot simply shows tracks for different simulations. No weighting by abundance has been applied, so only the range spanned by the simulations, not typical values, can be read from the plot.

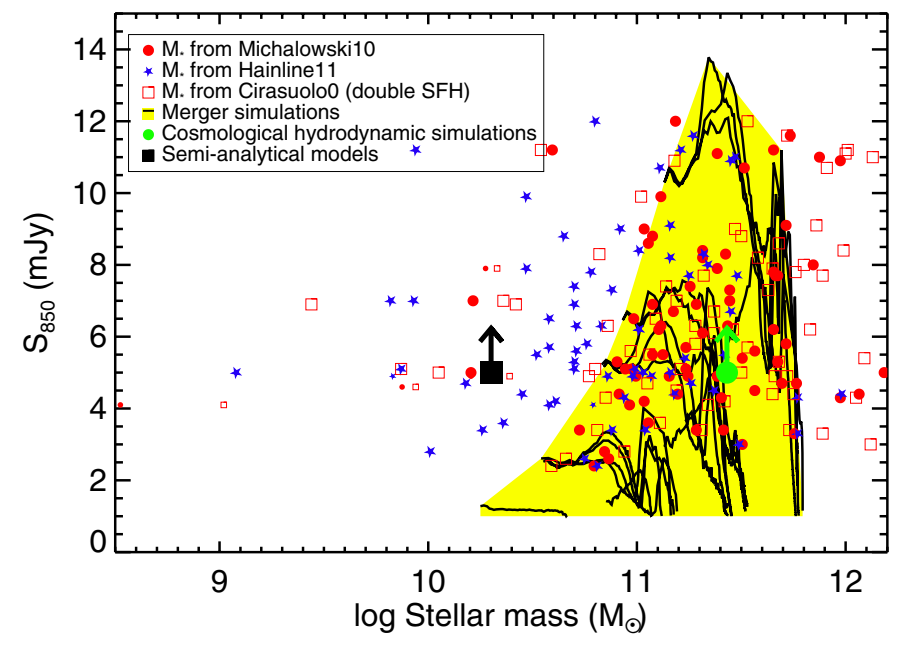

Fig. 4. Submillimetre flux densities as a function of the stellar masses of SMGs. Solid lines represent the evolutionary tracks from the merger hydrodynamic simulations (Narayanan et al. 2009, 2010a,b; Hayward et al. 2011a,b) enclosed in the yellow shaded area, the big square represents the median stellar mass of $2 \times 10^{10} M_{\odot}$ for SMGs with $S_{850}>$ $5 \mathrm{mJy}$ in the semi-analytical models (Baugh et al. 2005; Swinbank et al. 2008; González et al. 2011; Almeida et al. 2011), whereas the big circle corresponds to the median value derived by Davé et al. (2010) from the cosmological hydrodynamic simulations. Other symbols represent the observational data for SMGs with stellar masses from M10 (red circles), H11 (blue stars) and our new estimates based on C10 and double-component SFH (red squares). Small symbols indicate SMGs at $z<0.5$. According to the hydrodynamic simulations, many of the stellar masses derived by $\mathrm{H} 11$ are too low to result in measurable submillimetre flux, while the stellar masses derived by M10 are consistent with the hydrodynamic simulation predictions (within the observational errors). Neither the H11, M10 or C10 stellar masses appear to agree, on average, with the median stellar mass predicted by the semi-analytic models.

We note that in order to reach $S_{850 \mu \mathrm{m}} \gtrsim 5 \mathrm{mJy}$, these simulations require stellar mass to exceed $M_{*} \simeq 1 \times 10^{11} M_{\odot}$. As can be seen from Fig. 4, the predicted stellar mass range is in good agreement with the SMG stellar masses derived by M10. We note that, in the cold infall model of SMGs, comparably high stellar masses are also preferred (Davé et al. 2010, their Fig. 2).

On the other hand, the semi-analytical models of Baugh et al. (2005), Swinbank et al. (2008), González et al. (2011, Fig. 3) and Almeida et al. (2011) predict significantly lower stellar masses for SMGs, with a median $M_{*} \simeq 2 \times 10^{10} M_{\odot}$ (indicated by the square in Fig. 4). Such a value is lower even than the vast majority of the stellar masses derived by H11, confirming the findings of Swinbank et al. (2008) that the stellar masses of SMGs are generally systematically under-predicted by this class of semianalytic model.

\section{Specific star-formation rates}

One important step towards locating SMGs within the general framework of galaxy/star formation at high redshift is to establish whether, on average, they simply represent the most massive extension of the normal star-forming galaxy population in the young active Universe at $z>2$ (as predicted by Davé et al. 2010, in cosmological hydrodynamic simulations), or whether they are extreme outliers in terms of specific star-formation rate (sSFR).

To explore this we compare the properties of SMGs with other galaxies at similar redshifts as presented by Daddi et al. (2007, Fig. 17), Kajisawa et al. (2010, Fig. 2) and Rodighiero et al. (2011, Fig. 1). 
M. J. Michałowski et al.: The stellar masses and specific star-formation rates of submillimetre galaxies

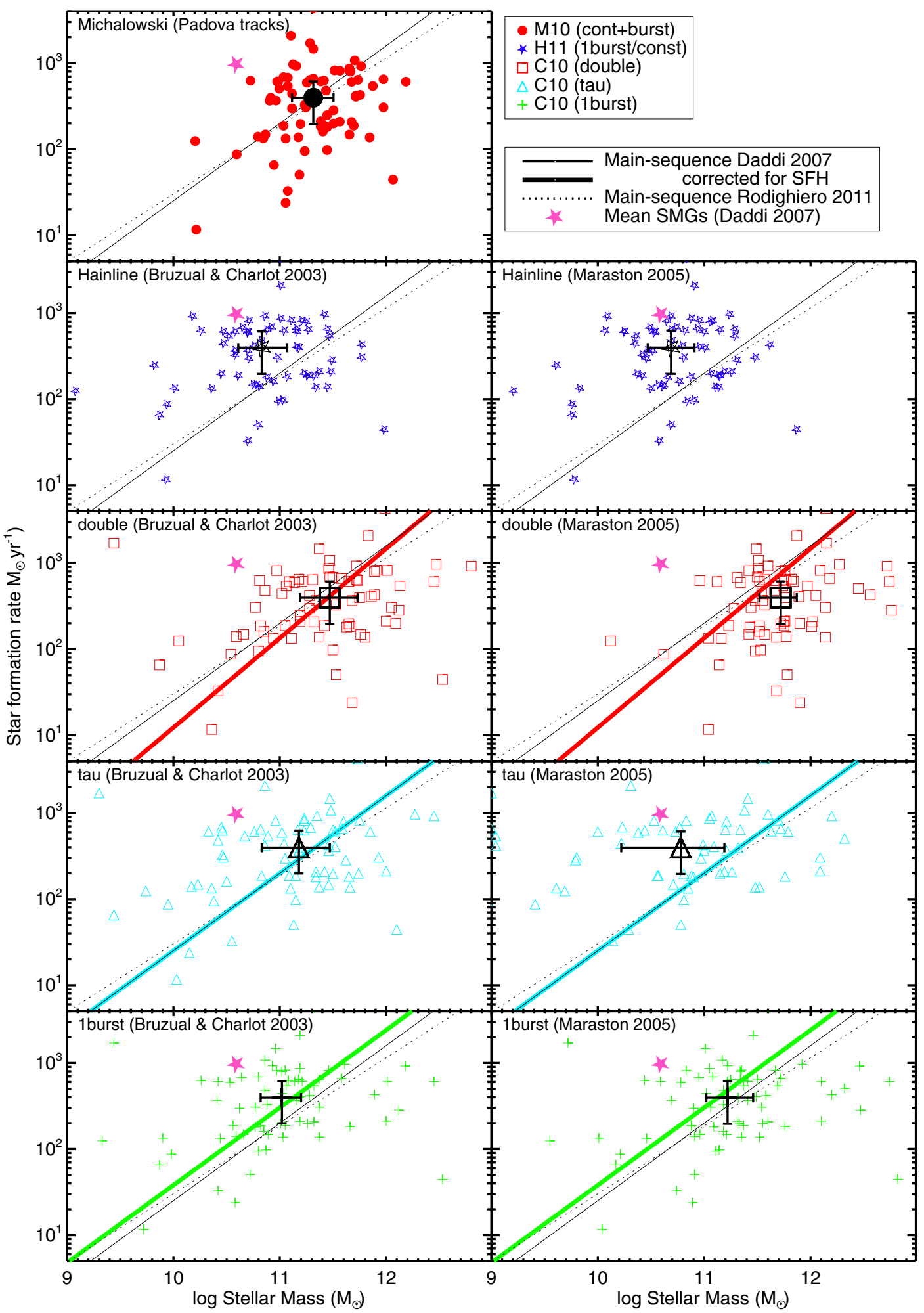

Fig. 5. SFRs of SMGs as a function of stellar masses derived using the stellar population synthesis models of Bruzual \& Charlot (2003, left) and Maraston (2005, right). The symbols correspond to the stellar masses derived by M10 (red circles), H11 (blue stars) and our new estimates based on the SED modelling method of Cirasuolo et al. $(2010, \mathrm{C} 10)$ for three alternative forms of star formation history (SFH): double-component model (red squares), exponentially-declining (tau) model (light blue triangles) and single burst (green pluses). The median values are shown as large black symbols with $3 \sigma$ error bars. The "main-sequence" of star-forming galaxies at $z \simeq 2$ is shown as a solid line (Daddi et al. 2007) and dotted line (Rodighiero et al. 2011). The thick, coloured solid lines represent the "main-sequence" of Daddi et al. (2007) at $z \simeq 2$ corrected for the systematic difference in average stellar mass introduced by the self-consistent adoption of SFH to model SMGs in each panel (see Sect. 4 for details). All results have been converted to the Chabrier (2003) IMF. Unless the exponentially declining (tau model) SFH with the Maraston (2005) models are assumed, the SMGs are completely consistent with the high-mass end of this sequence. This agreement is essentially perfect for the M10 and double-component models. The magenta star shows the position of an average SMG as plotted by Daddi et al. (2007). Clearly, regardless of the assumed evolutionary stellar model or SFH, this supposed typical value of sSFR for SMGs is in fact unrepresentative of real SMGs. 
For the SMGs we adopt the SFRs derived by M10 from the mid-far infrared emission. The comparison is shown in Fig. 5. Here, for the nine different determinations of SMG masses considered in this paper, we plot SFR versus $M_{*}$ for all the individual SMGs in the sample, and compare the results with the location of the so-called "main-sequence" of star-forming galaxies at $z \simeq 2$ (Daddi et al. 2007; Rodighiero et al. 2011).

In order to ensure that this comparison is fair and selfconsistent, we have also investigated how the choice of SFH influences the location of this "main-sequence". We assumed that the SFR estimate is independent of the adopted SFH (as it is usually derived from bolometric or monochromatic luminosity), and derived the stellar masses of all galaxies the UDS field in CANDELS (Grogin et al. 2011; Koekemoer et al. 2011) at $z=1.5-3$ and $M_{*}=10^{9.5}-10^{11.6} M_{\odot}$ (Cirasuolo et al., in prep.) using the same SED fitting method outlined in Sect. 2.2 for double-burst, single-burst and tau SFHs. If we adopt the results of the single-burst models for these galaxies, we obtained masses consistently lower by 0.1 dex relative to those derived using the tau models; in Fig. 5 ("1 burst" panels) this is indicated by the position of the coloured thick line towards lower stellar masses. On the other hand, as expected, the adoption of the double-burst models yields higher stellar masses. However, this shift transpires to be mass-dependent; at $10^{10} M_{\odot}$ the double-burst SFH produces stellar masses 0.3 dex higher than the tau SFH, whereas this shift is only $0.1 \mathrm{dex}$ at $10^{11.5} M_{\odot}$ (resulting in steepening of the thick line in the "double" panels). The latter is a significantly smaller shift than the 0.3 dex difference found between the double-burst and tau model stellar masses for SMGs (Table 1).

It is clear from this figure that, for almost all models the SMGs lie essentially exactly on this "main-sequence", within the errors. It is also clear that, even for the lower mass determinations, the average deviation from the "main-sequence" is relatively modest. As expected (given Table 1 and the discussion in Sect. 2.2), the offset is greatest for the exponentiallydecreasing tau SFH with the Maraston (2005) models, but even then it can be seen that the majority of the SMGs are still actually consistent with the "main-sequence", and the formal $\simeq 3 \sigma$ offset in average SSFR is in fact driven by a subset of extreme outliers. It can also be seen that none of the various determinations of SMG stellar masses yields a SSFR for a typical SMG as extreme as that claimed by Daddi et al. (2007). This discrepancy is likely because their stellar mass estimates are based on dynamical masses from high-excitation CO line observations (Tacconi et al. 2006), which may bias the stellar mass estimates low, as we explained in Sect. 3.1. Moreover, their use of infrared luminosity of $\sim 10^{13} L_{\odot}$, estimated from a single $850 \mu$ m detection (Greve et al. 2005; Tacconi et al. 2006), represents an overestimate, as revealed by recent Herschel studies (Magnelli et al. 2012; Roseboom et al. 2012, $\sim 10^{12.5-12.7} L_{\odot}$ ) making the SFR estimate of Daddi et al. (2007) a factor of 2-3 too high.

To quantify the apparent consistency between the properties of SMGs and other star-forming galaxies at comparable epochs we report, in Table 1, the mean and median sSFRs for the SMGs as derived from the various alternative models (as stated above these values are IMF-independent). Again, with the possible exception of the Maraston (2005) tau-model, all the median values are clearly consistent with the values of a few $\mathrm{Gyr}^{-1}$ found by Daddi et al. (2007) and Kajisawa et al. (2010). Hence, it would appear that, while a few SMGs may be caught at unusually spectacular moments, in general it seems hard to avoid the conclusion that the SMG population is perfectly consistent with the highmass end of the general star-forming galaxy population at these redshifts. Interestingly, it is only really the double-component models which yield mean and median values which are formally consistent with each other, with the other models always producing a few extreme outliers with very low stellar masses. Thus, the use of the most physically plausible star-formation histories yields consistent and fairly well defined typical mean sSFR values for SMGs of 2-3 Gyr ${ }^{-1}$, in rather good agreement with the height of the plateau in sSFR at $z>2$ discussed by González et al. (2010).

\section{Conclusions}

We have explored the potential origins of systematic differences in the stellar masses derived for SMGs, and have found that the difference between the stellar-mass estimates of M10 and H11 can be explained by the combination of different assumed IMFs, stellar population models and types of star formation histories. The available evidence supports the use of the Chabrier IMF (with the Salpeter IMF yielding excessive masses for several SMGs). The choice of which stellar synthesis model to adopt turns out to be relatively unimportant compared to the choice of star formation history. The one exception to this is the combination of an exponentially declining star formation history with the models of Maraston (2005), which produces radically different, unrealistically low stellar masses, presumably due to the very strong assumed TP-AGB contribution in these models.

For the same IMF and evolutionary synthesis model, the remaining difference between the average stellar mass estimates of M10 and H11 can be accounted for by differences in adopted star-formation history. Specifically, the two-component models of M10 yield values $\simeq 3$ times larger than the single-component models utilised by H11. We have explored this issue in more detail, by running further single-burst, tau-model and doublecomponent model fits using the method of $\mathrm{C} 10$ and using both the Bruzual \& Charlot (2003) and Maraston (2005) models. We find that the models which allow past and "present" starformation activity to be disconnected yield very consistent average stellar masses in the range $M_{*}=2-3 \times 10^{11} M_{\odot}$, regardless of precisely how this is parameterized. We argue, therefore, that the main reason that authors such as H11 and Wardlow et al. (2011) have concluded in favour of typical SMG stellar masses $M_{*}<1 \times 10^{11} M_{\odot}$ is that they have assumed models in which the SFR in SMGs was always as high or higher prior to the epoch at which the object has been selected on the basis of its extreme star-formation activity. The more physically realistic two-component models are supported by other evidence for rising star-formation histories in star-forming galaxies at $z>2$.

Considering potential orientation effects, and updated estimates of dynamical masses, we find that even the higher stellar mass estimates of M10 with the Chabrier IMF certainly cannot be excluded with current $\mathrm{CO}$ line data, and indeed yield total masses in good agreement with dynamical mass estimates. We also argue that SMG stellar masses $M_{*} \simeq 2 \times 10^{11} M_{\odot}$ appear to be perfectly plausible given current measurements of the evolving mass function of the general galaxy population selected at near-infrared and mid-infrared wavelengths.

We also show that the mass estimates of M10 are in good agreement with the predictions of hydrodynamical merger models of SMGs and the cold inflow scenario, whereas those of H11 are closer to (but still not as low as) the much lower-mass predictions of certain semi-analytic models of galaxy formation.

Finally, we find that the inferred specific star-formation rates of SMGs are perfectly consistent with those derived for other star-forming galaxies at comparable redshifts, suggesting that most SMGs simply represent the high-mass extension of the "main-sequence" of star-forming galaxies at $z \geq 2$, and in 
general should not be considered as outliers in the stellar massSFR relation.

Acknowledgements. We thank Joanna Baradziej and our anonymous referee for help with improving this paper and Georgios Magdis for comments. M.J.M. and J.S.D. acknowledge the support of the UK Science \& Technology Facilities Council. J.S.D. acknowledges the support of the Royal Society via a Wolfson Research Merit award, and the support of the European Research Council through an Advanced Grant. The Dark Cosmology Centre is funded by the Danish National Research Foundation. This research has made use of Tool for OPerations on Catalogues And Tables (TOPCAT; Taylor 2005): www . starlink . ac .uk/topcat/ and NASA's Astrophysics Data System Bibliographic Services.

\section{References}

Alexander, D. M., Bauer, F. E., Chapman, S. C., et al. 2005, ApJ, 632, 736 Alexander, D. M., Brandt, W. N., Smail, I., et al. 2008, AJ, 135, 1968 Almeida, C., Baugh, C. M., \& Lacey, C. G. 2011, MNRAS, 417, 2057 Alongi, M., Bertelli, G., Bressan, A., et al. 1993, A\&AS, 97, 851 Baugh, C. M., Lacey, C. G., Frenk, C. S., et al. 2005, MNRAS, 356, 1191 Berciano Alba, A., Koopmans, L. V. E., Garrett, M. A., Wucknitz, O., \& Limousin, M. 2010, A\&A, 509, A54

Bertelli, G., Bressan, A., Chiosi, C., Fagotto, F., \& Nasi E. 1994, A\&AS, 106, 275

Borys, C., Smail, I., Chapman, S. C., et al. 2005, ApJ, 635, 853 Bouché, N., Lehnert, M. D., \& Péroux, C. 2005, MNRAS, 364, 319 Bressan, A., Fagotto, F., Bertelli, G., \& Chiosi C. 1993, A\&AS, 100, 647 Bruzual, G., \& Charlot, S. 2003, MNRAS, 344, 1000

Bussmann, R. S., Dey, A., Armus, L., et al. 2012, ApJ, 744, 150

Cappellari, M., Bacon, R., Bureau, M., et al. 2006, MNRAS, 366, 1126 Caputi, K. I., Cirasuolo, M., Dunlop, J. S., et al. 2011, MNRAS, 413, 162 Chabrier, G. 2003, ApJ, 586, L133

Chapman, S. C., Blain, A. W., Smail, I., \& Ivison, R. J. 2005, ApJ, 622, 772 Cimatti, A., Cassata, P., Pozzetti, L., et al. 2008, A\&A, 482, 21

Cirasuolo, M., McLure, R. J., Dunlop, J. S., et al. 2007, MNRAS, 380, 585 Cirasuolo, M., McLure, R. J., Dunlop, J. S., et al. 2010, MNRAS, 401, 1166 Conroy, C., \& Gunn, J. E. 2010, ApJ, 712, 833

Coppin, K., Chapin, E. L., Mortier, A. M. J., et al. 2006, MNRAS, 372, 1621 Daddi, E., Dickinson, M., Morrison, G., et al. 2007, ApJ, 670, 156 Davé, R., Finlator, K., Oppenheimer, B. D., et al. 2010, MNRAS, 404, 1355 Downes, D., \& Solomon, P. M. 2003, ApJ, 582, 37 Drory, N., Bender, R., Feulner, G., et al. 2004, ApJ, 608, 742 Dunlop, J. S., McLure, R. J., Yamada, T., et al. 2004, MNRAS, 350, 769 Dye, S., Eales, S. A., Aretxaga, I., et al. 2008, MNRAS, 386, 1107 Dye, S., Eales, S., Moncelsi, L., \& Pascale, E. 2010, MNRAS, 407, L69 Eminian, C., Kauffmann, G., Charlot, S., et al. 2008, MNRAS, 384, 930 Engel, H., Tacconi, L. J., Davies, R. I., et al. 2010, ApJ, 724, 233 Erb, D. K., Steidel, C. C., Shapley, A. E., et al. 2006, ApJ, 646, 107 Fagotto, F., Bressan, A., Bertelli, G., \& Chiosi, C. 1994a, A\&AS, 104, 365 Fagotto, F., Bressan, A., Bertelli, G., \& Chiosi, C. 1994b, A\&AS, 105, 29 Fagotto, F., Bressan, A., Bertelli, G., \& Chiosi, C. 1994c, A\&AS, 105, 39 Finlator, K., Oppenheimer, B. D., \& Davé, R. 2011, MNRAS, 410, 1703 Fontanot F., Monaco P., Silva L., \& Grazian A. 2007, MNRAS, 382, 903 Genzel, R., Baker, A. J., Tacconi, L. J., et al. 2003, ApJ, 584, 633 Gerhard, O., Kronawitter, A., Saglia, R. P., \& Bender, R. 2001, AJ, 121, 1936 Girardi, L., Bressan, A., Chiosi, C., Bertelli, G., \& Nasi, E. 1996, A\&AS, 117, 113

Girardi, L., Bressan, A., Bertelli, G., \& Chiosi, C. 2000, A\&AS, 141, 371 González, V., Labbé, I., Bouwens, R. J., et al. 2010, ApJ, 713, 115 González, J. E., Lacey, C. G., Baugh, C. M., \& Frenk, C. S. 2011, MNRAS, 413, 749

Greve, T. R., Bertoldi, F., Smail, I., et al. 2005, MNRAS, 359, 1165 Grillo, C., Gobat, R., Lombardi, M., \& Rosati, P. 2009, A\&A, 501, 461 Grogin, N. A., Kocevski, D. D., Faber, S. M., et al. 2011, ApJS, 197, 35

Hainline, L. J. 2008, Multi-Wavelength Properties of Submillimeter-Selected Galaxies, Ph.D. Thesis, California Institute of Technology

Hainline, L. J., Blain, A. W., Smail, I., et al. 2009, ApJ, 699, 1610

Hainline, L. J., Blain, A. W., Smail, I., et al. 2011, ApJ, 740, 96 Hatsukade, B., Iono, D., Akiyama, T., et al. 2010, ApJ, 711, 974 Hayward, C. C., Kereš, D., Jonsson, P., et al. 2011a, ApJ, 743, 159

Hayward, C. C., Narayanan, D., Jonsson, P., et al. 2011b, ASP Conf. Ser., 440, 369

Hayward, C. C., Jonsson, P., Kereš, D., et al. 2012, MNRAS, submitted [arXiv: 1203.1318]
Henriques, B., Maraston, C., Monaco, P., et al. 2011, MNRAS, 415, 3571 Hezaveh, Y. D., \& Holder, G. P. 2011, ApJ, 734, 52

Iglesias-Páramo, J., Buat, V., Hernández-Fernández, J., et al. 2007, ApJ, 670, 279

Ikarashi, S., Kohno, K., Aguirre, J. E., et al. 2011, MNRAS, 415, 3081 Ivison, R. J., Smail, I., Le Borgne, J. F., et al. 1998, MNRAS, 298, 583 Ivison, R. J., Papadopoulos, P. P., Smail, I., et al. 2011, MNRAS, 412, 1913 Kajisawa, M., Ichikawa, T., Tanaka, I., et al. 2009, ApJ, 702, 1393

Kajisawa, M., Ichikawa, T., Yamada, T., et al. 2010, ApJ, 723, 129 Kneib, J., van der Werf, P. P., Kraiberg Knudsen, K., et al. 2004, MNRAS, 349, 1211

Knudsen, K. K., Kneib, J., Richard, J., Petitpas, G., \& Egami, E. 2010, ApJ, 709, 210

Koekemoer, A. M., Faber, S. M., Ferguson, H. C., et al. 2011, ApJS, 197, 36

Kriek, M., Labbé, I., Conroy, C., et al. 2010, ApJ, 722, L64

Kroupa, P. 2001, MNRAS, 322, 231

Lima, M., Jain, B., Devlin, M., \& Aguirre, J. 2010, ApJ, 717, L31

MacArthur, L. A., McDonald, M., Courteau, S., \& Jesús González, J. 2010, ApJ, 718, 768

Magnelli, B., Lutz, D., Santini, P., et al. 2012, A\&A, 539, A155

Maraston, C. 2005, MNRAS, 362, 799

Maraston, C., Daddi, E., Renzini, A., et al. 2006, ApJ, 652, 85

Maraston, C., Pforr, J., Renzini, A., et al. 2010, MNRAS, 407, 830

Menéndez-Delmestre, K., Blain, A. W., Alexander, D. M., et al. 2007, ApJ, 655, L65

Menéndez-Delmestre, K., Blain, A. W., Smail, I., et al. 2009, ApJ, 699, 667

Michałowski, M., Hjorth, J., \& Watson, D. 2010a, A\&A, 514, A67

Michałowski, M. J., Watson, D., \& Hjorth, J. 2010b, ApJ, 712, 942

Mosleh, M., Williams, R. J., Franx, M., \& Kriek, M. 2011, ApJ, 727, 5

Motohara, K., Takata, T., Iwamuro, F., et al. 2005, AJ, 129, 53

Murphy, E. J., Chary, R. R., Alexander, D. M., et al. 2009, ApJ, 698, 1380

Narayanan, D., Cox, T. J., Hayward, C. C., Younger, J. D., \& Hernquist, L. 2009, MNRAS, 400, 1919

Narayanan, D., Dey, A., Hayward, C. C., et al. 2010a, MNRAS, 407, 1701

Narayanan, D., Hayward, C. C., Cox, T. J., et al. 2010b, MNRAS, 401, 1613

Negrello, M., Hopwood, R., De Zotti, G., et al. 2010, Science, 330, 800

Noeske, K. G., Weiner, B. J., Faber, S. M., et al. 2007, ApJ, 660, L43

Paciga, G., Scott, D., \& Chapin, E. L. 2009, MNRAS, 395, 1153

Papovich C., Finkelstein S. L., Ferguson H. C., Lotz J. M., \& Giavalisco M. 2011, MNRAS, 412, 1123

Pope, A., Chary, R. R., Alexander, D. M., et al. 2008, ApJ, 675, 1171

Pozzetti, L., Bolzonella, M., Lamareille, F., et al. 2007, A\&A, 474, 443

Ricciardelli, E., Trujillo, I., Buitrago, F., \& Conselice, C. J. 2010, MNRAS, 406, 230

Riechers, D. A., Hodge, J., Walter, F., Carilli, C. L., \& Bertoldi, F. 2011, ApJ, 739, L31

Rodighiero, G., Daddi, E., Baronchelli, I., et al. 2011, ApJ, 739, L40

Roseboom, I. G., Ivison, R. J., Greve, T. R., et al. 2012, MNRAS, 419, 2758

Salpeter, E. E. 1955, ApJ, 121, 161

Santini, P., Maiolino, R., Magnelli, B., et al. 2010, A\&A, 518, L154

Schael, A. M. 2009, The Star-Formation History of Massive Galaxies, Ph.D. Thesis, University of Edinburgh

Silva, L., Granato, G. L., Bressan, A., \& Danese, L. 1998, ApJ, 509, 103 Swinbank, A. M., Lacey, C. G., Smail, I., et al. 2008, MNRAS, 391, 420 Swinbank, A. M., Smail, I., Chapman, S. C., et al. 2010a, MNRAS, 405, 234

Swinbank, A. M., Smail, I., Longmore, S., et al. 2010b, Nature, 464, 733

Tacconi, L. J., Neri, R., Chapman, S. C., et al. 2006, ApJ, 640, 228

Tamura, Y., Iono, D., Wilner, D. J., et al. 2010, ApJ, 724, 1270

Targett, T. A., Dunlop, J. S., McLure, R. J., et al. 2011, MNRAS, 412, 295

Taylor, M. B. 2005, in Astronomical Data Analysis Software and Systems XIV, ed. P. Shopbell, M. Britton, \& R. Ebert, ASP Conf. Ser., 347, 29

Thomson, M. G., \& Chary, R. R. 2011, ApJ, 731, 72

Valiante, E., Lutz, D., Sturm, E., et al. 2007, ApJ, 660, 1060

van der Wel, A., Franx, M., van Dokkum, P. G., et al. 2006a, ApJ, 636, L21

van der Wel, A., Franx, M., Wuyts, S., et al. 2006b, ApJ, 652, 97

van Dokkum, P. G. 2008, ApJ, 674, 29

van Dokkum, P. G., \& Conroy, C. 2010, Nature, 468, 940

van Dokkum, P. G., \& Conroy, C. 2011, ApJ, 735, L13

Vieira, J. D., Crawford, T. M., Switzer, E. R., et al. 2010, ApJ, 719, 763

Wardlow, J. L., Smail, I., Coppin, K. E. K., et al. 2011, MNRAS, 415, 1479

Watabe, Y., Risaliti, G., Salvati, M., et al. 2009, MNRAS, 396, L1

Weidner, C., Kroupa, P., \& Pflamm-Altenburg, J. 2011, MNRAS, 412, 979

Wilson, G. W., Hughes, D. H., Aretxaga, I., et al. 2008, MNRAS, 390, 1061

Zibetti, S., Charlot, S., \& Rix, H. 2009, MNRAS, 400, 1181

Zibetti, S., Gallazzi, A., Charlot, S., Pasquali, A., \& Pierini, D. 2012, [arXiv: 1203.4571$]$ 
A\&A 541, A85 (2012)

Appendix A: Table with all stellar mass estimates

Table A.1. Stellar masses of individual SMGs calculated using various methods, stellar population models and star formation histories.

\begin{tabular}{|c|c|c|c|c|c|c|c|c|c|c|}
\hline \multirow[b]{4}{*}{ SMG } & \multirow[b]{4}{*}{$z$} & \multicolumn{9}{|c|}{ 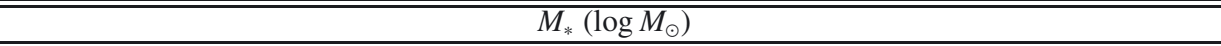 } \\
\hline & & ${\mathrm{M} 10^{a}}^{a}$ & $\mathrm{H} 11^{b}$ & $\mathrm{H} 11^{c}$ & $\mathrm{C} 10^{d}$ & $\mathrm{C} 10^{e}$ & $\mathrm{C} 10^{f}$ & $\mathrm{C} 10^{g}$ & $\mathrm{C} 10^{h}$ & $\mathrm{C} 10^{i}$ \\
\hline & & Padova & $\mathrm{BC} 03$ & M05 & $\mathrm{BC} 03$ & $\mathrm{BC} 03$ & $\mathrm{BC} 03$ & M05 & M05 & M05 \\
\hline & & double & single & single & 1burst & tau & 2burst & 1burst & tau & 2burst \\
\hline SMMJ030226.17+000624.5 & 0.080 & 10.275 & -99.000 & -99.000 & 10.010 & 10.000 & 10.330 & 9.810 & 9.810 & 10.590 \\
\hline SMMJ030227.73+000653.5 & 1.408 & 10.915 & 11.180 & 11.009 & 11.180 & 11.190 & 11.750 & 11.320 & 11.320 & 11.770 \\
\hline SMMJ030231.81+001031.3 & 1.316 & 10.205 & 9.080 & 9.209 & 9.330 & 9.740 & 10.050 & 9.550 & 9.690 & 10.120 \\
\hline SMMJ030236.15+000817.1 & 2.435 & 11.285 & 10.880 & 10.689 & 11.180 & 11.170 & 11.310 & 11.250 & 11.200 & 11.570 \\
\hline SMMJ030238.62+001106.3 & 0.276 & 8.525 & 10.790 & 10.639 & 8.220 & 8.520 & 9.020 & 8.520 & 7.200 & 9.480 \\
\hline SMMJ030244.82+000632.3 & 0.176 & 10.205 & 9.830 & 9.559 & 10.100 & 10.090 & 10.390 & 9.950 & 9.950 & 10.410 \\
\hline SMMJ105151.69+572636.0 & 1.147 & 11.175 & 11.450 & 11.109 & 11.370 & 11.370 & 11.370 & 11.520 & 11.520 & 12.140 \\
\hline SMMJ105155.47+572312.7 & 2.686 & 11.235 & 10.580 & 10.419 & 10.880 & 10.460 & 11.530 & 11.180 & 8.650 & 11.570 \\
\hline SMMJ105158.02+571800.2 & 2.239 & 11.675 & 11.480 & 11.259 & 11.320 & 11.340 & 11.320 & 11.600 & 11.260 & 12.020 \\
\hline SMMJ105200.22+572420.2 & 0.689 & 10.945 & 9.870 & 9.759 & 9.870 & 9.440 & 9.870 & 10.170 & 7.630 & 11.140 \\
\hline SMMJ105201.25+572445.7 & 2.148 & 11.115 & 10.470 & 10.349 & 11.020 & 11.040 & 11.020 & 11.020 & 10.220 & 11.540 \\
\hline SMMJ105207.49+571904.0 & 2.689 & 11.655 & & 10.879 & 11.190 & 11.230 & 11.460 & 11.050 & 11.050 & 11.510 \\
\hline $.79+571906.4$ & 2.372 & 11.385 & 11.070 & 10.879 & 11.660 & 11.650 & 11.660 & 11.760 & 11.760 & 12.010 \\
\hline SMMJ10 & 2.142 & 11.565 & 11.370 & 11.189 & 11.190 & 11.420 & 11.190 & 11.310 & 11.190 & 11.750 \\
\hline SMMJ10 & 1.956 & 11.445 & 9.820 & 9.609 & -99.000 & -99.000 & 11.190 & -99.000 & -99.000 & -99.000 \\
\hline $30.73+572209.5$ & 2.611 & 11.875 & 11.470 & 11.289 & 11.320 & 10.890 & 12.130 & 11.690 & 9.040 & 11.970 \\
\hline SMMJ105238.19+571651.1 & 1.852 & 10.905 & 10.700 & 10.519 & 11.160 & 11.460 & 11.680 & 11.410 & 11.210 & 11.480 \\
\hline SMMJ105238.30+572435.8 & 3.036 & 11.975 & 11.450 & 11.289 & 11.180 & 10.820 & 11.180 & 11.500 & 9.000 & 11.820 \\
\hline SMMJ123549.44+621536.8 & 2.203 & 11.425 & 11.320 & 11.149 & 10.820 & 10.830 & 10.820 & 10.880 & 10.880 & 11.520 \\
\hline $3.26+621337.7$ & 2.098 & 11.075 & 10.650 & 10.489 & 10.640 & 11.290 & 11.500 & 10.950 & 10.770 & 11.310 \\
\hline $14+620901.7$ & 1.875 & 11.505 & 11.230 & 11.069 & 11.260 & 11.740 & 12.090 & 11.550 & 11.150 & 11.900 \\
\hline SMMJ123600.10+620253.5 & 2.710 & 11.285 & -99.000 & -99.000 & 9.440 & 9.300 & 9.440 & 9.720 & 9.000 & 12.150 \\
\hline SMMJ123600.15+621047.2 & 1.994 & 11.385 & 10.470 & 10.249 & 11.140 & 11.520 & 11.650 & 11.410 & 11.630 & 11.840 \\
\hline SMMJ123606.72+621550.7 & 2.416 & & & & & 11.500 & & 11.330 & 10.810 & 11.720 \\
\hline MJ123606.85+621021.4 & 2.509 & 11.735 & 11.270 & 11.149 & & 11.500 & 11.720 & 11.340 & 11.340 & 11.750 \\
\hline MJ123616.15+621513.7 & 2.578 & 11.715 & 10.760 & 10.799 & & 10.860 & 11.280 & 11.580 & 9.040 & 12.050 \\
\hline & 1.86 & & & & & & & .090 & 10.900 & 11.430 \\
\hline 8.4 & 1.988 & .655 & 0.780 & 10.619 & 10.890 & 11.460 & 60 & 11.210 & 10.710 & 11.570 \\
\hline 29.7 & 2.466 & 665 & 11.250 & 11.059 & 11.020 & 11.600 & 11.890 & 11.340 & 11.210 & 11.720 \\
\hline $.13+621045.8$ & 1.013 & 11.445 & 11.030 & 10.909 & 10.870 & 11.150 & 11.500 & 11.140 & 10.810 & 11.530 \\
\hline $632.61+620800.1$ & 1.993 & 11.075 & 10.520 & 10.359 & 11.060 & 10.670 & 11.060 & 11.370 & 8.830 & 11.740 \\
\hline SMMJ123634.51+621241.0 & 1.219 & 11.405 & 10.940 & 10.769 & 10.850 & 10.500 & 10.850 & 11.180 & 8.610 & 11.520 \\
\hline SMMJ123635.59+621424.1 & 2.005 & 11.125 & 11.420 & 11.239 & 11.610 & 12.270 & 12.470 & 11.920 & 11.600 & 12.270 \\
\hline SMMJ123636.75+621156.1 & 0.557 & 10.215 & 9.930 & 9.779 & & 10.030 & 10.360 & 10.040 & 8.480 & 11.040 \\
\hline SMMJ123651.76+621221.3 & 0.298 & 9.875 & -99.000 & -99.000 & 9.430 & 9.590 & 9.940 & 9.510 & 8.650 & 10.230 \\
\hline SMMJ123707.21+621408.1 & 2.484 & 11.695 & 11.260 & 11.139 & 11.050 & 11.090 & 11.050 & 11.370 & 10.570 & 11.750 \\
\hline SMMJ123711.98+621325.7 & 1.992 & 11.035 & 10.610 & 10.419 & 10.560 & 11.150 & 11.420 & 10.880 & 10.560 & 11.230 \\
\hline SMMJ123712.05+621212.3 & 2.914 & 11.845 & 11.340 & 11.139 & 11.030 & 11.660 & 11.800 & 11.350 & 11.480 & 11.680 \\
\hline SMMJ123716.01+620323.3 & 2.037 & 11.675 & -99.000 & -99.000 & 12.010 & 11.850 & 13.150 & 12.100 & 10.000 & 13.100 \\
\hline & 0.979 & & 10.800 & 10.689 & & 11.130 & & 10.910 & 10.780 & 11.780 \\
\hline & & & & & & 60 & & .460 & 10.310 & 11.870 \\
\hline SM & 1.544 & & & & & 10.470 & & 11.060 & 9.800 & 11.440 \\
\hline+424422.5 & 2.805 & .565 & 10.710 & 10.649 & 10.970 & 10.960 & 10.970 & 10.830 & 10.830 & 11.420 \\
\hline SMMJ131215.27+423900.9 & 2.565 & 12.065 & 11.980 & 11.869 & 12.530 & 12.100 & 12.530 & 12.820 & 10.290 & 13.220 \\
\hline SMMJ131222.35+423814.1 & 2.565 & 11.505 & 11.490 & 11.309 & 12.120 & 11.620 & 12.120 & 12.470 & 9.790 & 12.760 \\
\hline SMMJ131225.20+424344.5 & 1.038 & 10.795 & 10.810 & 10.609 & 10.590 & 10.170 & 10.590 & 10.910 & 8.340 & 11.770 \\
\hline SMMJ131225.73+423941.4 & 1.554 & 10.965 & 10.580 & 10.469 & 10.410 & 11.180 & 11.340 & 10.690 & 11.000 & 11.550 \\
\hline SMMJ131228.30+424454.8 & 2.931 & 11.415 & 11.040 & 10.819 & 11.080 & 11.230 & 11.730 & 11.370 & 10.610 & 11.770 \\
\hline SMMJ131231.07+424609.0 & 2.713 & 10.995 & -99.000 & -99.000 & 15.890 & 15.330 & 13.360 & 12.320 & 12.320 & 12.450 \\
\hline SMMJ131232.31+423949.5 & 2.320 & 11.765 & 10.180 & 10.089 & 12.180 & 12.450 & 12.800 & 12.460 & 12.200 & 12.720 \\
\hline SMMJ131239.14+424155.7 & 2.242 & 11.255 & 10.700 & 10.529 & 10.530 & 11.020 & 11.140 & 10.780 & 10.380 & 11.340 \\
\hline SMMJ141741.81+522823.0 & 1.150 & 11.755 & 11.770 & 11.619 & 11.890 & 11.460 & 11.890 & 12.200 & 10.990 & 12.560 \\
\hline SMMJ141742.04+523025.7 & 0.661 & 10.865 & 10.750 & 10.569 & 10.660 & 10.230 & 10.660 & 10.960 & 8.410 & 11.420 \\
\hline SMMJ141750.50+523101.0 & 2.128 & 10.845 & 10.010 & 9.829 & 9.900 & 10.360 & 10.940 & 10.010 & 9.610 & 11.040 \\
\hline SMMJ141800.40+512820.3 & 1.913 & 12.185 & 11.160 & 10.999 & 12.450 & 13.620 & 12.450 & 12.740 & 11.790 & 12.740 \\
\hline SMMJ141802.87+523011.1 & 2.127 & 10.725 & 10.260 & 10.069 & 10.260 & 10.460 & 10.810 & 10.440 & 10.440 & 10.960 \\
\hline SMMJ141809.00+522803.8 & 2.712 & 11.975 & 11.770 & 11.479 & 11.370 & 11.930 & 12.050 & 11.540 & 12.090 & 12.140 \\
\hline SMMJ141813.54+522923.4 & 3.484 & 11.055 & 10.360 & 10.499 & 10.440 & 11.100 & 11.110 & 10.460 & 10.850 & 11.160 \\
\hline
\end{tabular}


Table A.1. continued.

\begin{tabular}{|c|c|c|c|c|c|c|c|c|c|c|}
\hline \multirow[b]{4}{*}{ SMG } & \multirow[b]{4}{*}{$z$} & \multicolumn{9}{|c|}{$M_{*}\left(\log M_{\odot}\right)$} \\
\hline & & ${\mathrm{M} 10^{a}}$ & $\mathrm{H} 11^{b}$ & $\mathrm{H} 11^{c}$ & $\mathrm{C} 10^{d}$ & $\mathrm{C} 10^{e}$ & $\mathrm{C} 10^{f}$ & $\mathrm{C} 10^{g}$ & $\mathrm{C} 10^{h}$ & $\mathrm{C} 10^{i}$ \\
\hline & & Padova & $\mathrm{BC} 03$ & M05 & ВC03 & ВC03 & BC03 & M05 & M05 & M05 \\
\hline & & double & single & single & 1burst & tau & 2burst & 1burst & tau & 2 burst \\
\hline SMMJ163627.94+405811.2 & 3.180 & 10.985 & 10.580 & 10.249 & 10.420 & 10.760 & 11.090 & 10.590 & 10.730 & 11.120 \\
\hline SMMJ163631.47+405546.9 & 2.283 & 11.435 & 10.830 & 10.699 & 10.860 & 10.420 & 10.860 & 11.180 & 8.610 & 11.660 \\
\hline SMMJ163639.01+405635.9 & 1.495 & 10.975 & 10.700 & 10.539 & 11.210 & 10.330 & 11.210 & 11.220 & 8.510 & 11.520 \\
\hline SMMJ163650.43+405734.5 & 2.378 & 11.315 & 11.160 & 10.939 & 11.580 & 11.590 & 11.580 & 11.720 & 11.720 & 12.180 \\
\hline SMMJ163658.19+410523.8 & 2.454 & 11.515 & 11.110 & 10.959 & 11.050 & 11.750 & 11.910 & 11.350 & 11.700 & 11.760 \\
\hline SMMJ163658.78+405728.1 & 1.190 & 11.235 & 10.990 & 10.839 & 10.800 & 10.380 & 10.800 & 11.110 & 8.960 & 11.480 \\
\hline SMMJ163704.34+410530.3 & 0.840 & 10.595 & 9.940 & 9.759 & 9.980 & 9.950 & 10.540 & 10.210 & 9.410 & 10.620 \\
\hline SMMJ163706.51+405313.8 & 2.374 & 11.655 & 11.210 & 11.079 & 11.460 & 11.230 & 12.010 & 11.670 & 10.530 & 12.090 \\
\hline SMMJ221724.69+001242.1 & 0.510 & 11.055 & -99.000 & -99.000 & 10.580 & 10.150 & 11.680 & 10.890 & 8.340 & 11.900 \\
\hline SMMJ221725.97+001238.9 & 3.094 & 11.705 & -99.000 & -99.000 & 10.860 & 11.480 & 11.470 & 11.170 & 11.380 & 11.510 \\
\hline SMMJ221733.02+000906.0 & 0.926 & 11.385 & -99.000 & -99.000 & 12.000 & 12.000 & 12.000 & 12.100 & 12.090 & 11.760 \\
\hline SMMJ221733.12+001120.2 & 0.652 & 11.075 & 10.700 & 10.579 & 10.420 & 10.550 & 10.420 & 10.740 & 10.140 & 11.680 \\
\hline SMMJ221733.91+001352.1 & 2.555 & 11.715 & 11.160 & 10.959 & 11.200 & 11.520 & 11.860 & 11.490 & 11.090 & 11.890 \\
\hline SMMJ221735.15+001537.2 & 3.098 & 11.115 & 10.710 & 10.459 & 10.620 & 11.050 & 11.280 & 10.900 & 10.970 & 11.280 \\
\hline SMMJ221735.84+001558.9 & 3.089 & 11.155 & 10.860 & 10.689 & 10.930 & 11.350 & 11.720 & 11.220 & 11.070 & 11.600 \\
\hline SMMJ221737.39+001025.1 & 2.614 & 11.315 & -99.000 & -99.000 & 10.960 & 11.470 & 11.370 & 11.010 & 11.410 & 11.490 \\
\hline SMMJ221804.42+002154.4 & 2.517 & 11.035 & 10.920 & 10.739 & 10.800 & 10.450 & 11.470 & 11.080 & 10.230 & 11.500 \\
\hline SMMJ221806.77+001245.7 & 3.623 & 11.315 & 11.010 & 10.839 & 11.330 & 11.730 & 11.990 & 11.600 & 11.570 & 12.140 \\
\hline
\end{tabular}

Notes. The Chabrier (2003) IMF is assumed. -99 indicates the lack of an estimate for a given SMG. ${ }^{(a)}$ Stellar mass derived by M10. ${ }^{(b)}$ Stellar mass derived by $\mathrm{H} 11$ with BC03 models. ${ }^{(c)}$ Stellar mass derived by H11 with M05 models. ${ }^{(d)}$ Stellar mass derived as in C10 with BC03 models and single-burst SFH. ${ }^{(e)}$ Stellar mass derived as in C10 with BC03 models and tau SFH. ${ }^{(f)}$ Stellar mass derived as in C10 with BC03 models and double-burst SFH. ${ }^{(g)}$ Stellar mass derived as in C10 with M05 models and single-burst SFH. ${ }^{(h)}$ Stellar mass derived as in C10 with M05 models and tau SFH. ${ }^{(i)}$ Stellar mass derived as in C10 with M05 models and double-burst SFH. References: M10 (Michałowski et al. $2010 \mathrm{a}$ ); H11 (Hainline et al. 2011); C10 (Cirasuolo et al. 2010); BC03 (Bruzual \& Charlot 2003); M05 (Maraston 2005). 